\title{
Strutture ad anfore: un sistema di bonifica dei suoli. Qualche parallelo dalle Provinciae Hispanicae
}

\section{Embedded-amphore structures: a way to reclaim lands. Some parallel from Provinciae Hispanicae}

\author{
MARIAVITTORIA ANTICO GALLINA
}

\section{RIASSUNTO}

L'autrice, partendo dallo studio dei fattori tecnici necessari per analizzare un singolare metodo costruttivo con anfore poste nel sottosuolo, illustra alcuni casi dell'Italia Cisalpina, come Mediolanum, Cremona, Novaria ed altri ancora e li mette a confronto con casi delle città ispane. La finalità degli accumuli di anfore si comprende attraverso le caratteristiche litologiche dei terreni: si tratta infatti di metodi di miglioramento delle condizioni geotecniche e idrauliche per i quali non è corretto usare come termine convenzionale «drenaggio». Sulla base di queste esperienze l'A. riflette sui casi di Saragozza e Cadice, e sulla terminologia più adeguata.

\section{RESUMEN}

La autora, que desde los años ochenta estudia los sistemas de mejora de las características del terreno con ánforas, partiendo del estudio de los factores técnicos necesarios para analizar un método único de construcción a base de ánforas colocadas en el suelo, ilustra algunos casos de la Italia Cisalpina, como Mediolanum, Cremona, Novaria y otros, y los compara con hallazgos en ciudades hispanas como Zaragoza y Cádiz. El propósito de los acúmulos de ánforas puede entenderse por las características litológicas de las zonas en las que se aplicó esta técnica, por lo que la autora reflexiona sobre la terminología más adecuada a aplicar según el objetivo perseguido por los técnicos que adoptaron esta particular forma de construcción.

\section{SUMMARY}

The author, who has been studying systems of reclaiming land using embedded-amphorae since the $80 \mathrm{~s}$, illustrates cases from Italia Cispalina, such as Mediolanum Cremona, Parma and Novaria among others and compares them with finds in Spanish cities (Zaragoza, Empuries, Cádiz) based on the study of the technical factors needed to analyze this particular building method. The purpose of these embedded-amphorae can be understood through the lithological soil characteristics of the areas in which this technique was used in order to improve the geotechnical and hydraulic soil conditions. The author reflects upon the most adequate terminology to apply based on the objectives of the technicians who applied this particular building style.

PAROLE CHIAVE: Interventi geotecnici e idraulici, età romana, anfore nel terreno, terminologia.

PALABRAS CLAVE: Mejora de las características del terreno, edad romana, ánforas, terminología.

KEY WORDS: Geotechnical and Hydraulic Reclaim, Roman Age, Embedded Amphorae, Terminology .

A Enrico, mio marito 1947-2008

Se al noto studioso spagnolo Miguel Beltrán Lloris si deve, oltre alla preziosa classificazione tipologica alla quale chi studia il materiale anforario ha fatto e fa ricorso, il merito di aver dilatato la sua analisi inserendo, nel quadro di una più generale casistica sull'uso secondario —oggi diremmo reimpiego o, più spietatamente, riciclaggio- di tali contenitori da trasporto, quello relativo all'ambito edilizio, credo che, per questo specifico settore, si debba riconoscere alla penisola italica il primato delle attestazioni archeologiche, e dunque il primato 'quantitativo' di questo singolare tipo di 'uso edilizio' delle anfore. ${ }^{1}$

\footnotetext{
${ }^{1}$ Basti considerare che già solo entro i 2000 mq. indagati all'interno dell'Università Cattolica di Milano - suburbio occidentale della città romana- sono state individuate svariate decine di accumuli anforari, oggetto di una tesi svolta nell'ambito della Scuola di Specializzazione in Archeologia del nostro Ateneo. Vd. già al riguardo Salsarola, Locatelli et alii 2001: 10 .
} 
Occupandomi, dalla metà degli anni $' 80,{ }^{2}$ di tale questione, ritengo utile da una parte rimarcare l'apporto dello studioso spagnolo e dall'altra riproporre alcuni, emblematici, casi italici e ispanici.

In effetti, sulla scorta in parte delle fonti letterarie, in parte dei rinvenimenti ${ }^{3}$ avvenuti anche al di fuori della penisola iberica, accanto ad anfore usate come urne funerarie, o per la decantazione di acqua salina, come vasi acustici, vasi per fiori, elementi interrati per definire confini, custodie per tesoretti monetali, Beltran Lloris pone le anfore nella loro qualità di materiale da costruzione. E' anzi proprio il contesto costruttivo che viene considerato per primo nel quadro delle tipologie applicative delle anfore quale materiale 'alternativo', usate intere o frammentate: si rammenta dunque il sistema di 'riempimento' delle coperture a cupola,${ }^{4} \mathrm{o}$ dei muri pertinenti a forni, ${ }^{5}$ le si menziona come elementi atti ad opere di contenimento (ByrsaCartagine), ${ }^{6}$ come condotte idriche, si osserva infine il caso di anfore che presentano dei fori all'altezza del collo, del ventre o nel peduncolo. Questo ultimo particolare denunzia il fatto che rispetto all'elencazione dei vari usi delle anfore così come possiamo già leggere nelle fonti letterarie ${ }^{7}$ Beltran Lloris mosse un ulteriore passo verso una lettura più critica delle condizioni di questo tipo di reperto, sebbene riconoscesse che «aunque desconocemos el uso exacto». ${ }^{8}$

Per il caso di anfore inserite nel terreno egli ricorda il riempimento di fossati, ${ }^{9}$ il più significativo

\footnotetext{
${ }^{2}$ Avendo introdotto già dai primi anni d'insegnamento i contenuti delle Scienze della terra come supporto agli argomenti trattati. L'interesse per il tema dei sistemi fondazionali e, fra essi, delle anfore nel terreno rientra pienamente nell'ottica interdisciplinare della Topografia antica, insegnata dalla scrivente nei corsi di laurea della Facoltà di Lettere e Filosofia e nella Scuola di Specializzazione in Archeologia dell'Università Cattolica del Sacro Cuore, sede di Milano. E', d'altronde, ampiamente risaputo che soprattutto per gli studi di Topografia antica l'esigenza di lettura ed interpretazione dei fenomeni antropici (insediamenti, necropoli, suddivisioni agrarie, opere infrastrutturali, sistemi di distribuzione e approvvigionamento idrico, tecniche e scelte edilizie) procede parallelamente al1'acquisizione dei dati ambientali ed alla valutazione delle peculiarità geomorfologiche e pedologiche dei territori.

${ }^{3}$ Già Morel 1877, s.v. Amphora, riportava il ritrovamento (1789) di un magazzino colmo d'anfore che colpisce perché morfologicamente accostabile alle strutture ad anfore oggetto di questa nota. La totale perdita di ogni dato rende impossibile ogni considerazione.

${ }^{4}$ Lugli 1957: 671-672.

${ }^{5}$ Con evidente effetto coibentante. Vd. anche Antico Gallina 2011c.

${ }^{6}$ Delattre 1894: 89-119.

${ }^{7}$ Nessuna fonte fa menzione della bonifica attuata con accumuli anforari. Per una breve sintesi vd. Antico Gallina 1996a: 68-70.

${ }^{8}$ Beltrán Lloris 1977: 584. Per una possibile motivazione vd. Antico Gallina 1996a: 85.

${ }^{9}$ Facendo riferimento al rinvenimento descritto da Dressel 1879: 129, 131.
}

dei quali è italico, avvenuto nel quartiere di Castro Pretorio, in Roma (1878). Dressel, che pubblicò il ritrovamento avvenuto fra Porta Collina e Porta Viminale, ${ }^{10}$ specificò trattarsi di centinaia di anfore individuate alla profondità di $\mathrm{m} \mathrm{1,40} \mathrm{dal} \mathrm{piano} \mathrm{di}$ campagna, per un'estensione, da $\mathrm{N}$ a $\mathrm{S}$, di m 16, capovolte e in più ordini sovrapposti, da 3 a 6 . Un «grande deposito», che doveva continuare verso $\mathrm{N}$, a motivo della grande quantità di anfore analogamente disposte individuate nel 1870, e il cui intento era, secondo Dressel, di riempimento della vicina fossa aggeris serviana e di isolamento dall'umidità. La datazione del tipo anforario più recente al $45 \mathrm{~d}$. C. e la sua posizione negli strati più bassi dell'accumulo consentirono già a Dressel l'ipotesi di un'unica realizzazione della colmata della fossa alla metà del I sec. d. C. ${ }^{11}$

Si ritornerà a breve sul soggetto del Castro Pretorio e su una panoramica italica ed ispanica a confronto, non prima, però, di aver tratteggiato taluni problemi a mio parere ancora connessi allo studio delle anfore nell'edilizia. Sarà l'occasione per una comune riflessione sulle numerose e diverse questioni che lo studio del loro 'uso secondario' comporta, dall' aspetto interpretativo a quello terminologico.

\section{LO STUDIO DELLE ANFORE NEI TERRENI}

\section{SCEGLIERE LA TERMINOLOGIA}

I numerosi esempi di ritrovamenti di analoga morfologia (anfore capovolte e verticali poste entro il terreno) nelle regioni meridionali della Gallia Transalpina $^{12}$ hanno condotto gli studiosi francesi all'adozione dell'espressione «vides sanitaires», intendendo definirne la precipua funzione, così come gli studiosi italiani hanno adottato per i numerosissimi casi della Gallia Cisalpina, accanto alla più generale indicazione di «deposito di anfore», il termine «drenaggio». La scelta di comprendere e verificare le modalità del supposto «drenaggio» (quale acqua, cioè, potessero e dovessero drenare) ha accompagnato i miei studi, consentendomi di ottenere alcune certezze. Analizzando le svariate centinaia di accumuli anforari, nel frattempo censite, secondo due criteri prevalenti:

${ }^{10}$ Fra via Gaeta, Volturno, Montebello, la zona dell'antica vigna della Certosa: Dressel 1879: 5. Vd. CAR 1977, III G, 13-14, via Montebello-Calatafimi.

${ }^{11}$ Dressel 1879: 194-195; Beltrán Lloris 1977: 392, nota 847.

${ }^{12}$ Soprattutto nel settore dell'ampia foce del fiume Rodano, per cui vd.i numerosi contributi di Laubenheimer . 

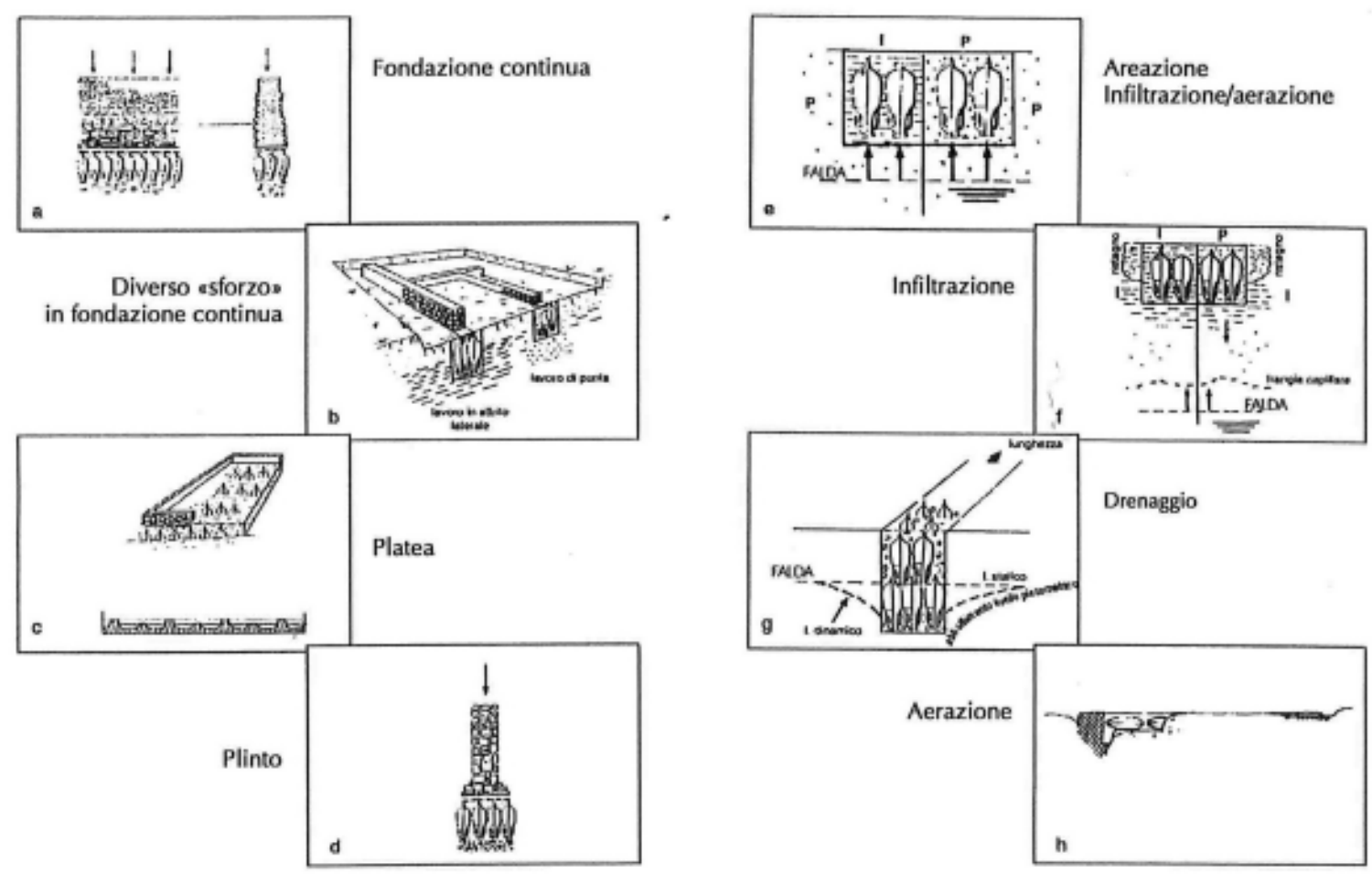

Figura 1. Le possibili funzioni assolte dalle strutture ad anfore: $\mathrm{a}-\mathrm{d}=$ bonifica geotecnica; $\mathrm{e}-\mathrm{h}=$ bonifica idraulica (da Antico Gallina, 1996a, elaborata).

a- quello delle analogie e delle diversificazioni fra gli accumuli, per ampiezza, forma, ubicazione, rapporti stratigrafici;

b- quello della stretta relazione fra accumulo e caratteri del terreno in cui esso si trovava, una prima convinzione fu l'inopportuna adozione generalizzata del termine «drenaggio».

In verità le convinzioni acquisite sono di vario ordine di fattori, ma possono sintetizzarsi in quattro punti che andrò, mano a mano, a motivare:

1) l'importanza di adottare una metodica di scavo d'équipe particolarmente attenta alla lettura delle peculiarità fisico-meccaniche del terreno interessato dall' accumulo anforario, da interpretare secondo i criteri univoci della geologia applicata ed i parametri della moderna geotecnica, nonché alla lettura dei materiali a contatto con le anfore componenti l'accumulo; ${ }^{13}$

2) la necessità di abbandonare la pericolosa definizione «drenaggio», non accettabile nemmeno

${ }^{13} \mathrm{Si}$ intende, ovviamente, qualunque tipo di materiale, sia esterno che interno alle anfore, per la cui descrizione è necessario fare riferimento a tutte le caratteristiche dei terreni, come colore, granulometria, presenza di incrostazioni carbonatiche, di materiali organici, ecc. Vd. al riguardo Alemani 2008: 12-19. come termine convenzionale o come sinonimo di 'deposito di anfore', o di usarla solo nei casi, rarissimi, in cui sia realmente attestata la funzione drenante, cioè l'allontanamento dell'eccedenza della componente liquida che saturava il terreno;

3) il riconoscimento delle concentrazioni d'anfore $^{14}$ come interventi di bonifica geotecnica o idraulica ante litteram;

4) la conseguente urgenza di individuare terminologie appropriate, più realisticamente rispondenti alle diverse funzioni assolte dagli accumuli anforari.

L'esito ultimo degli studi personalmente condot$\mathrm{ti}^{15}$ è stato dunque quello di proporre alla valutazione della comunità scientifica più allargata un primo termine 'alternativo', tale da non andare a incidere così pesantemente e così direttamente sull' aspetto interpretativo dell' accumulo anforario ('accumulo anforario', dunque, in luogo di 'deposito di anfore'), così come, a mio avviso, capitava e capita ogni volta che si adotta la definizione «drenaggio»: il termi-

\footnotetext{
${ }^{14}$ Qualora non sia indubitabilmente provato che si tratti di effettivi stoccaggi di contenitori alimentari.

${ }^{15}$ Illustrati nel 1995 in occasione del Convegno Bonifiche e drenaggi (cfr. Antico Gallina 1996a: 67-112) ed in altri articoli citati nella Bibliografia finale a questo saggio.
} 


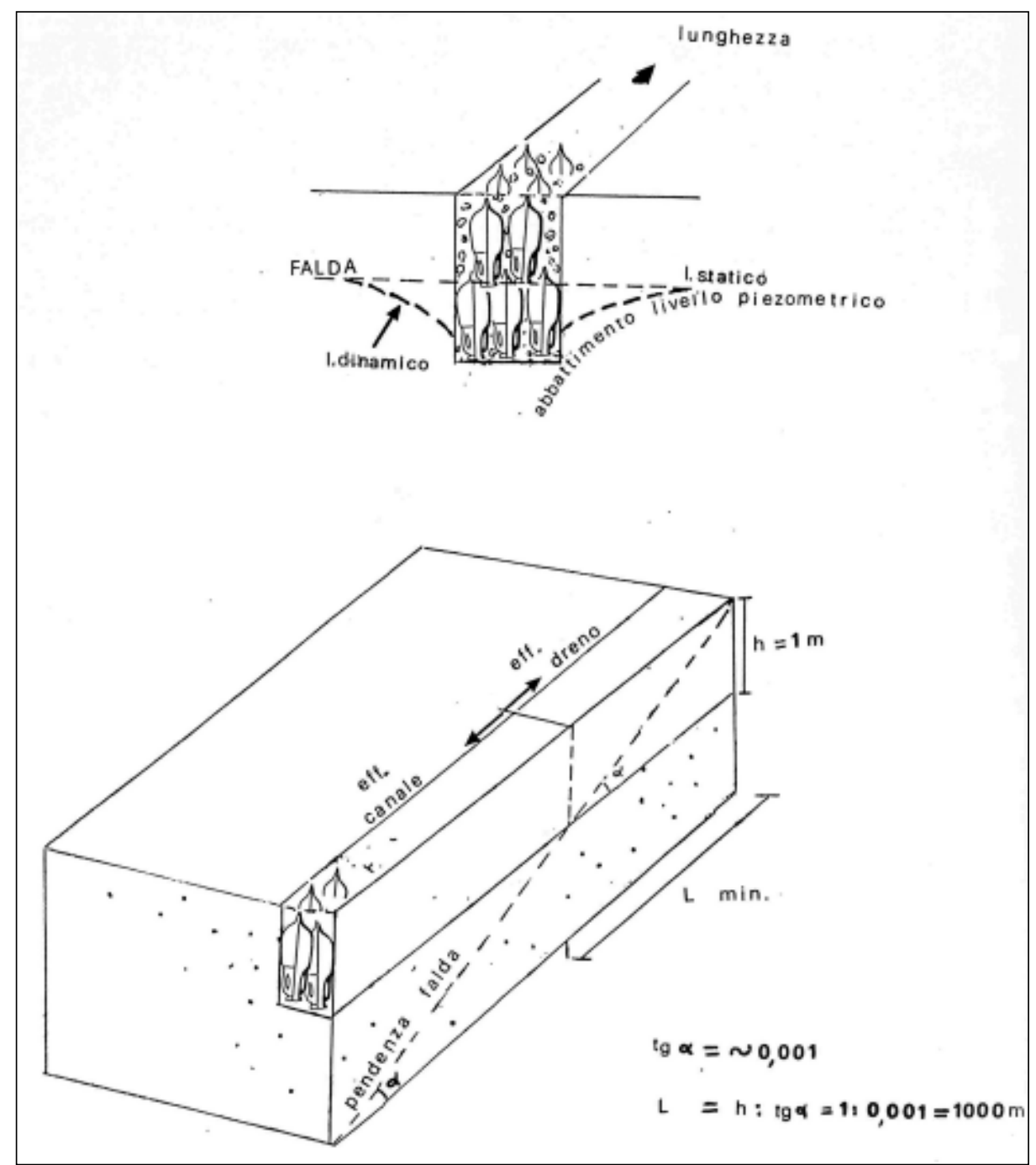

Figura 2. L'abbattimento del livello piezometrico necessita di un dreno proporzionale al gradiente piezometrico di falda, alla pendenza del suolo, all'entità di abbassamento desiderato (da Antico Gallina, 1996a).

ne proposto è, dunque, 'sistema ad anfore', ma potrebbe anche essere quello di 'struttura ad anfore'. La ragione sta nel fatto che l'accumulo di anfore rappresenta un 'sistema' volontario di intervento modificatorio rispetto a una situazione del suolo ritenuta non soddisfacente (quindi era una bonifica), non idonea in relazione al tipo di azione che l'uomo mise in atto su quello stesso suolo (edificio/ strada/ spazio aperto/ area calpestabile/ arginatura/ ecc.), e contestualmente esso costituiva una vera e propria 'struttura', adottata con precise finalità.

Ho considerato utile iniziare questa mia nota procedendo dal punto di arrivo degli studi effettuati da chi scrive, poiché la terminologia che d'ora innanzi verrà usata rappresenta il necessario punto di partenza delle valutazioni tecniche utili per comprendere e definire la funzione o le funzioni di volta in volta assolte dagli accumuli anforari (Fig. 1).

Con ciò non si intende certo negare l'adozione del termine 'drenaggio'. Se infatti, tra le finalità, possiamo effettivamente riconoscere alla struttura quella di favorire 'fisicamente' e per gravità l'allontanamento della componente liquida, consentito dal rapporto fra grado di pendenza del suolo, gradiente piezometrico, profondità e estensione della falda freatica, $\mathrm{o}$-nel caso queste condizioni non fossero soddisfa- 
centi all'uopo- dalla presenza di un dreno adeguato $^{16}$ (via di deflusso), potremo a buon diritto adottare il termine 'drenaggio' (Fig. 2). Mancando le condizioni sopra indicate ritengo, personalmente, - come spesso ribadito ${ }^{17}$ - si debba rinunciarvi e sostituirlo con un più generico 'accumulo di anfore' o 'sistema ad anfore' o ancora 'struttura ad anfore'.

A seconda poi dei caratteri dei singoli casi, avremo la possibilità di specificare ulteriormente con un termine che ne vada a definire la funzione: ad esempio, 'struttura di infiltrazione' o 'di aerazione' o 'di isolamento termico', o 'di fondazione' o 'di consolidamento', od altro ancora, fermo restando che, quando essa vada a migliorare la qualità del terreno, rientri sempre nella categoria dell'opera di bonifica.

\section{COMPRENDERE LE FINALITÀ}

Un problema persistente nello studio degli accumuli — dalle relazioni di scavo alla pubblicazione dello stesso- è il fatto che assai raramente è possibile a posteriori capirne la finalità secondo quei parametri che si considerano essenziali e sintetizzati nel precedente punto 1 ). Mi riferisco, cioè, alla natura 'certa' del materiale (terreno e/o altro) che si trova in posizione laterale, sottostante, interstiziale, soprastante rispetto all'insieme anforario (Fig. 3), e quindi ad una lettura che sia attendibile, da correlare con le opere (muri, pavimenti, cortili, ecc.) realizzate nel contesto dell'accumulo, oltre che alle quote di inizio e fine accumulo (Fig. 4). Per tale ragione ci si trova frequentemente nella necessità di 'immaginare' delle sezioni e di procedere nella ricerca delle finalità di tali strutture per 'deduzione' e per 'induzione'.

Posto, cioè, il dato di fatto che il sistema ad anfore risponda a una scelta 'intenzionale' da parte del costruttore, le motivazioni della scelta stanno nella volontà di modificare una condizione ambientale naturale deficitaria con l'inserimento della struttura ad anfore. Le uniche spie possibili della condizione

\footnotetext{
${ }^{16}$ Come si rileva nella Fig. 2 1'effetto 'dreno', sulla base delle caratteristiche del suolo e della falda potrebbe essere raggiunto - per l'abbatimento di n. 1 - con una struttura ad anfore lineare lunga m 1000 o con un condotto di pari lunghezza. Ciò sta a dimostrare che nei terreni con analoghe caratteristiche di pendenza e di falda la sola presenza di una struttura ad anfore di ridotto sviluppo (qualche metro) nella pratica non è assolutamente sufficiente ad abbattere il livello piezometrico e di conseguenza la struttura 'non è un drenaggio', non funziona da drenaggio, ma assolve altri compiti.

${ }^{17} \mathrm{Vd}$. nota 15.
}
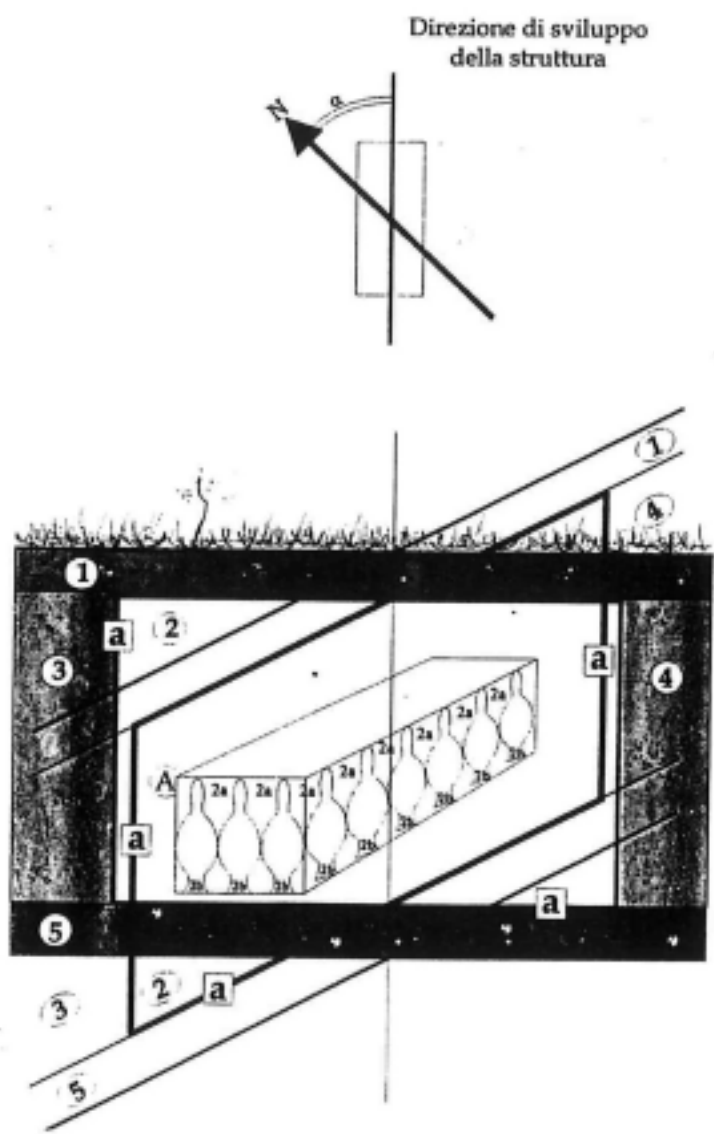

Figura 3. Visualizzazione schematica di una struttura ad anfore A, secondo la lettura delle sezioni longitudinale e trasversale. $\mathrm{a}=$ taglio; 2 = terreno laterale + riempimento a contatto con A; 2a materiali interstiziali; $2 \mathrm{~b}=$ materiali entro le anfore; 3, $4=$ materiali di contatto con 2; $5=$ terreno sottostante (elaborazione Antico Gallina).

naturale insoddisfacente, a parte una più generale conoscenza dei caratteri geomorfologici e idraulici del territorio in oggetto, sono quelle della tipologia di terreno a contatto con l'accumulo. Non è certo il caso di rimarcare che, ad esempio, la presenza di componenti argillose o torbose attorno alle anfore non si concilia affatto con la funzione 'drenante', per il loro noto comportamento in presenza di acqua ipogea (rigonfiamento); né si concilia se le stesse componenti si trovano negli spazi fra le anfore (interstizi): due casi per i quali il sistema si presta ad essere letto non certo come elemento drenante, ma come bonifica geotecnica di suoli la cui capacità di portanza sia stata - o possa essere- compromessa dalla presenza di acqua sotterranea. Qualora trovassimo negli interstizi materiali permeabili (permeabili all'aria, come ghiaia, frammenti laterizi, ceramici ecc.) la 


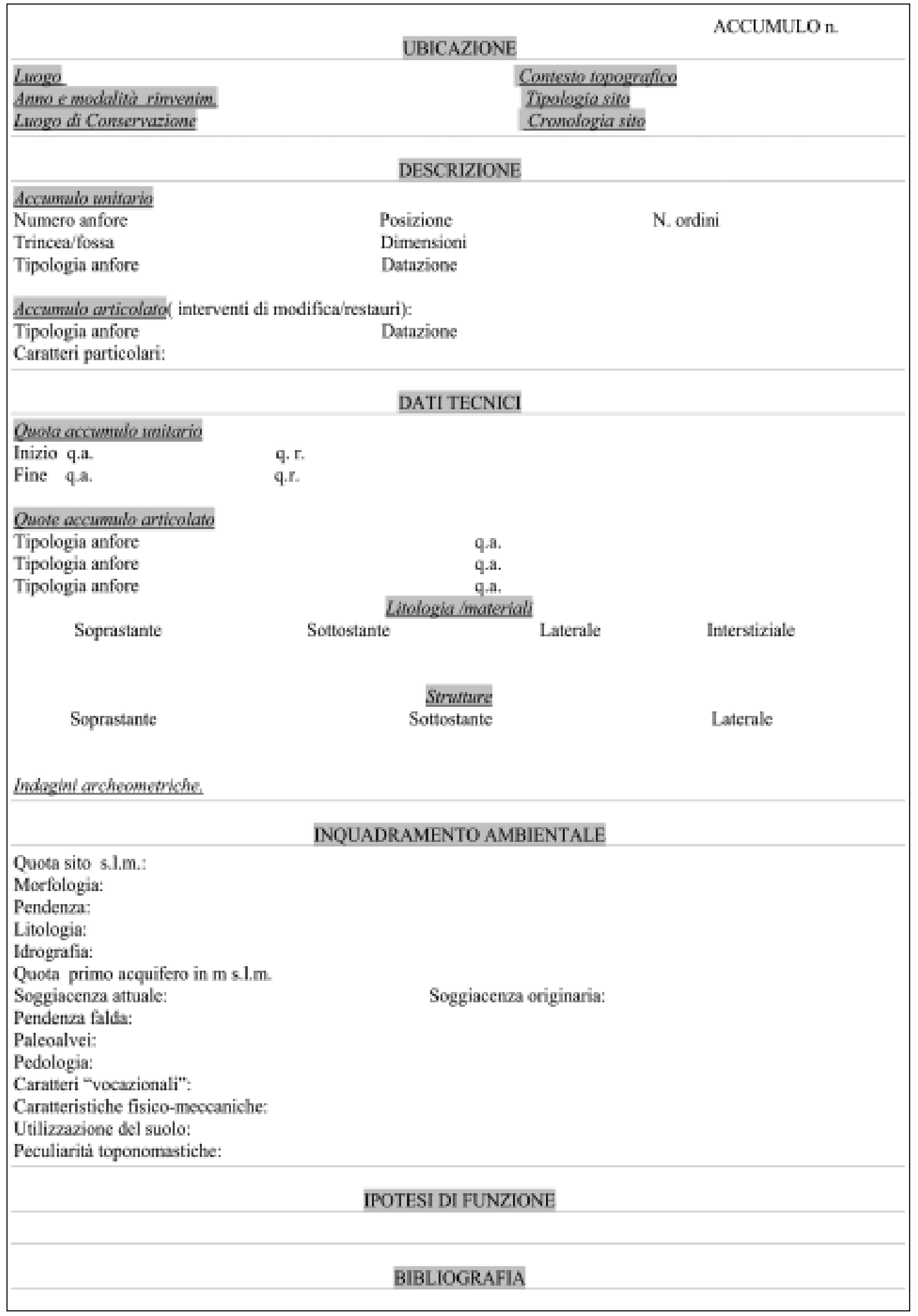

Figura 4. Tipo di Scheda 'sintetica' proponibile per il censimento di un accumulo (da Antico Gallina 2008). 
struttura può aver assolto la funzione di aerazione per la salubrità dell' opera soprastante. ${ }^{18}$

Appare, di fatto, generalizzata la metodica dei sistemi ad anfore in tutti quei suoli che si trovino in condizione di imbibizione o in stato, temporaneo o persistente, di saturazione, a motivo di litologie - diffuse o circoscritte, ma frequenti- contrarie alla percolazione delle acque, come lo sono quelle argillose o torbose.

\section{ALCUNI CASI DALLA PENISOLA ITALICA}

Un minimo assaggio, a questo punto, delle centinaia di casi italici, scelto fra quelli meglio documentati, prima di una lettura propositiva di qualche caso ispanico, relativo all'antica Tarraconensis ed alla Baetica.

STRUTTURE DI BONIFICA CON FUNZIONE DI CONSOLIDAMENTO E DI FONDAZIONE

E', sicuramente, la finalità più utilizzata, secondo i crismi della bonifica geotecnica.

Un esempio italico assai 'scenografico' può essere il percorso - di cui sono superstiti m 21, per una larghezza di $\mathrm{m} 6^{19}$ - rinvenuto nel suburbium $\mathrm{W}$ di Cremona (via Massarotti), in un settore sfiorato in età romana dal passaggio del fiume Po. A m 5 di profondità, sul duplice ordine sovrapposto di anfore, verticali, uno spesso strato di terra di riporto ricca di materiali di scarto uniformava, compattandola, la quota dei puntali anforari, per l'appoggio di sesquipedali posti di piatto: una lastricatura semplice, con un apparato di fondazione funzionale al miglioramento delle peculiarità di un terreno scadente, cedevole, per un percorso adducente al fiume Po (Fig. 5 ab). La datazione delle anfore alla metà del I sec. d. C. è compatibile con le opere di ricostruzione urbana promossa da Vespasiano dopo la distruzione ottoniana. ${ }^{20}$ Analoga situazione fu individuata fra la vicina via Amidani-Bissolati, ove i camminamenti così realizzati permettevano il transito entro un' area compromessa dalla presenza di buche di scarico di materiali edilizi e di resti di attività di macellazione, altre

\footnotetext{
$18 \mathrm{Vd}$. per questi ed altri esempi di correlazioni Antico Gallina 1996a: 84-88.

${ }^{19} \mathrm{Il}$ percorso subì i danni causati da edificazioni medievali, fra cui la cinta muraria.

${ }^{20}$ Passi Pitcher 1994: 149; 1998a: 100, 109 e fig. 21. Vd. inoltre Volontè 1998: 304-305, ove si data l'accumulo, sulla base delle tipologie anforarie, alla fine del i sec. d. C., con rari riutilizzi di anfore della seconda metà del i sec. a. C.
}

condizioni di scadimento delle capacità di portanza del terreno. ${ }^{21}$ Si noti che la fascia periurbana più a ridosso dell' antico alveo padano e quindi più umida ha restituito vari accumuli anforari di bonifica e che gli accumuli qui ricordati si collocano nelle depressioni che il modello altimetrico digitale della città ha ben messo in rilievo. ${ }^{22}$

Ulteriore caso. La massicciata in ghiaia di un troncone della strada Parma-Brixellum che, staccandosi dal cardine massimo alla periferia $\mathrm{N}$ di Parma (tra via Cuneo e via Palermo), proseguiva lungo la riva di un corso d'acqua canalizzato (Canale Naviglio), correva lungo una estesa struttura di centinaia di anfore, poste coricate e intercalate a pali (fascia laterale destinata al transito pedonale?), che con una disposizione che definirei a pettine si estendevano parzialmente al di sotto della massicciata $\operatorname{stessa}^{23}$ (Fig. 6 ab). Altri tratti della stessa infrastruttura, con sottofondo di anfore, furono rinvenuti più in prossimità della città (v.le Mentana-Borgo del Naviglio; via Benedetta; via Trieste). ${ }^{24}$ Siamo al cospetto di un unico fronte di bonifica esteso per $\mathrm{Km} 2$ ca., di oltre 20.000 anfore, ${ }^{25}$ che si imponeva come opera di consolidamento di un terreno scuro, quindi organico, e saturo ( tuttora paludoso), di ridotta portanza. E', in effetti, l'area dell'alluvium antico, i cui suoli si presentano da sempre argillosi con alterazioni lenticolari surtumose nere e sabbioso-ghiaiose della media pianura.

Ad Altino, oltre che nell' area dell'abitato anche nei pressi delle grandi strade extraurbane ove si collocano le aree funerarie sono attestate numerose strutture ad anfore (fine I sec. a. C. - fine I sec. d. C.), di tipologia varia (brindisine, Lamboglia 2, Dr. 6a e 6b, Dr. $7 / 11$ e 28 , Dr. 2/4), ma con morfologia analoga (corpi oblunghi). ${ }^{26} \mathrm{La}$ frequenza degli accumuli, la loro modesta consistenza, $\mathrm{i}$ terreni presenti, torbosi e organici in un contesto dall'assetto idrografico instabile, talune situazioni di collasso di strutture tombali, ne consentono una lettura che orienta verso cir-

${ }^{21}$ Bishop, Mariotti 1985: 116-118; Passi Pitcher 1998 b: 133; Mariotti 1998: 291-292.

${ }^{22}$ Passi Pitcher 2003: 135

${ }^{23}$ Non concordo con la finalità di «drenaggio» attribuita all'opera anforaria dall' autrice della notizia Calvani Marini 2006: 190-191 (rappresenta il collegamento fra Parma e il suo porto fluviale; vd. anche Ead., 1998: 239), e ribadita in Barbieri, Manzella 2006: 208 e 318.

${ }^{24}$ Dall'Aglio 1992: 181 .

${ }^{25}$ Calvani Marini 1998: 239; Catarsi Dall’Aglio 1998: 253 254.

${ }^{26}$ Gli interventi, per i quali vd. Tirelli, Balista et alii 1988 , sono considerati «drenaggi» e accettati secondo tale accezione anche in lavori recenti come Cecchini, Ridolfi 2010: nota 9 . 

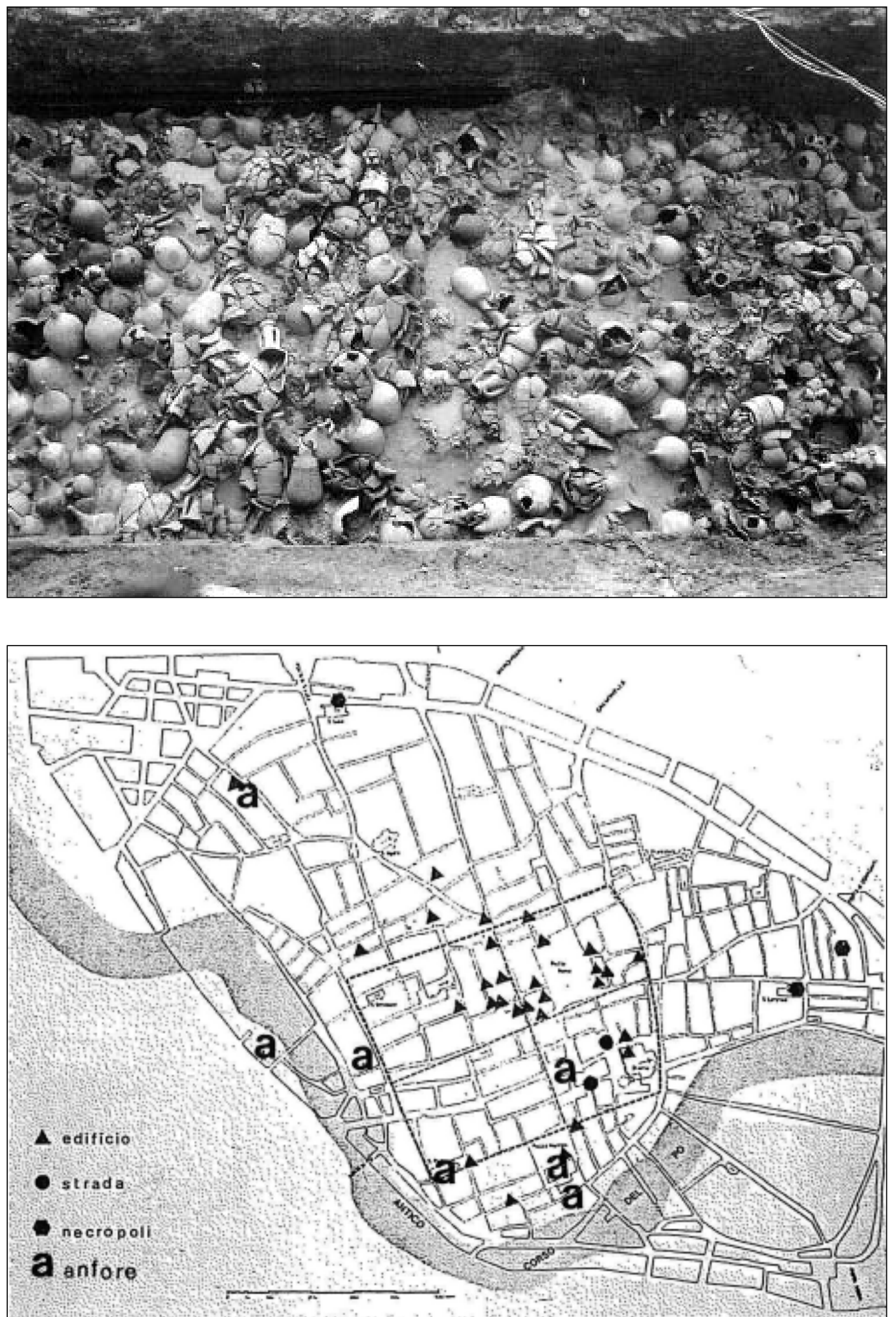

Figura 5 a-b. Cremona, via Massarotti e la posizione degli accumuli anforari (da Passi Pitcher 1998 a, rielaborata). 

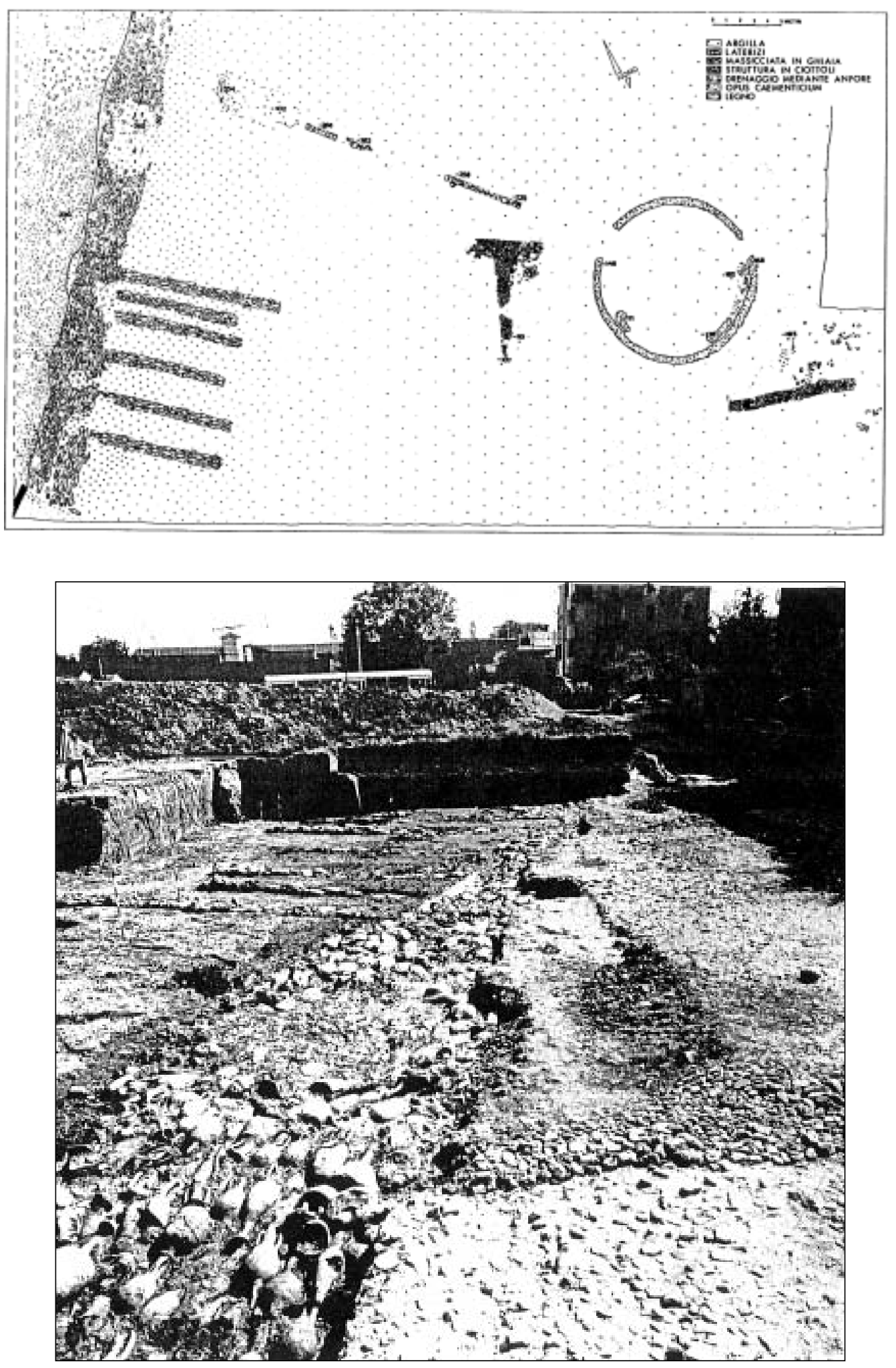

Figura 6 a-b. Parma, l'accumulo di via Palermo (da Calvani Marini 1992, modificato). 


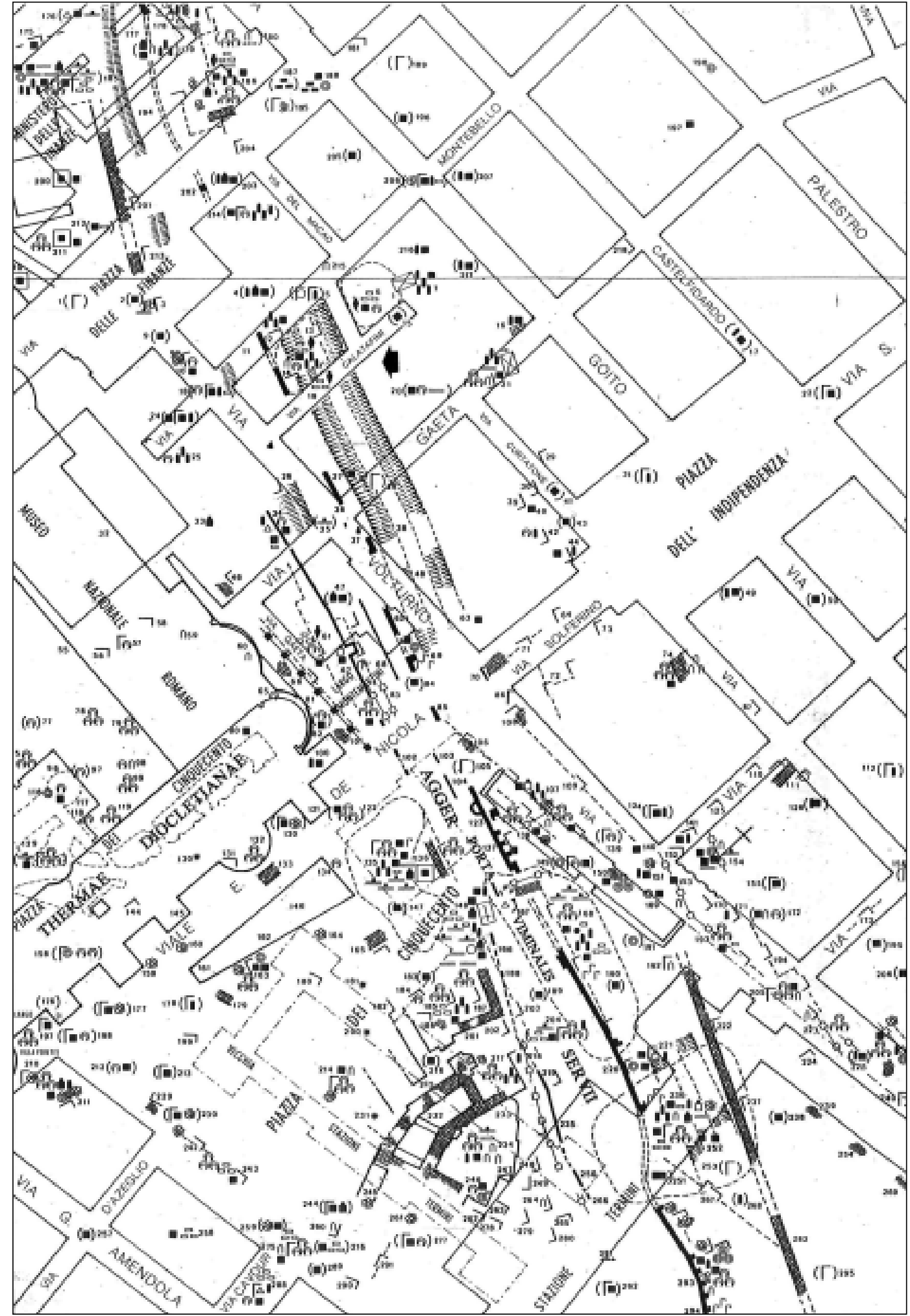

Figura 7. I ritrovamenti di strutture ad anfore in corrispondenza della fossa aggeris, Roma (stralcio da CAR, 1977). 
coscritti interventi di costipamento degli avvenuti cedimenti o di sostegno del terreno, quale forma preventiva a cedimenti o ripiegamenti di manufatti sopra terra.

Riprendendo, ora, il caso del riempimento della fossa aggeris serviana citato anche da Beltran Lloris, la verifica delle peculiarità litologiche in corrispondenza della zona del ritrovamento, completamente pianeggiante e corrispondente al «lato più debole della città», ${ }^{27} \mathrm{mi}$ porta a puntualizzare, con la presenza di un substrato composto da un'alternanza di tufi terrosi, scorie, detriti lavici, pomici e livelli lacustri su depositi da colata piroclastica, ${ }^{28}$ quelle caratteristiche di disgregabilità, di permeabilità e fessurabilità, atte a renderlo ricettore di sedimenti e detriti (eolici, argillosi, organici), cui può imputarsi la scarsa capacità di opporsi a spinte soprastanti o laterali (Fig. 7).

La situazione topografica riecheggia quella degli accumuli (fine I sec. a. C. - I sec. d. C.) rinvenuti a Novaria. Essi ben si conciliano con l'adozione del termine munitio o della definizione munire moenia/ viam, ${ }^{29}$ che recano in sé il significato di 'rinforzare': distribuiti — seppur con una discontinuità che potrebbe anche imputarsi a carenza o perdita di ulteriori ritrovamenti- lungo la cinta tardorepubblicana e all'interno di essa, ${ }^{30}$ paiono, sulla base di una precisa correlazione con le peculiarità del terreno, ricondursi ad opere di camminamento o di sostegno onde evitare lo slittamento e il ripiegamento verso la città del potente muro di cinta. Il terreno, caratterizzato dalla presenza di lenti argillose e soggetto a ristagni di acqua, giustifica dunque gli accumuli, concentrati là dove la realtà degli impaludamenti è posta in evidenza anche dall'uso di strutture fondanti a pali, ${ }^{31}$ mentre la quota alla quale furono posti in opera gli accumuli, all'altezza delle fondazioni delle mura, è coerente con l'ipotesi di una funzione atta a contenere cedimenti del suolo e il ripiegamento delle mura per cedimento, ma atta contemporaneamente, a mio avviso, a consolidare quella fascia interna alla cinta, libera da costruzioni, che poteva esser utile per la manutenzione dell'opera muraria e di conseguenza per il passaggio di carri e di materiali ${ }^{32}$ (Fig. 8).

\footnotetext{
${ }^{27}$ Coarelli 1984: 20.

${ }^{28}$ Marra, Rosa 2004: Tav. 9, Unità 7.

${ }^{29}$ Rimando al riguardo ad Antico Gallina 2008c

${ }^{30}$ Gli accumuli sono stati individuati lungo Baluardo Q. Sella ( 5 siti), in via Giulietti, via Passalacqua: cfr. Antico Gallina 1996a: 74-75; Tra terra e acque: 378- 380, 386, 403.

${ }^{31}$ Tra terra e acque: 379.

${ }^{32}$ Sono queste le deduzioni che appaiono nella tesi di laurea di F. EPIFANI, Novaria: gli accumuli anforari. Una verifica dei criteri di «bonifica» adottati in età romana, Università Cattolica del Sacro Cuore, Milano, a.a. 2004-05, relatore
}

In parte assimilabili le finalità dell'accumulo anforario dell'Urbe, poiché esso oltre che isolare dall'umidità capillare sostituiva il terreno naturale asportato per la creazione del terrapieno, riempiendo l'ampia superficie dell'avvallamento largo m 36

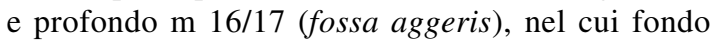
doveva affiorare il velo dell'acquifero superiore. ${ }^{33}$ L'accumulo è stato in effetti rinvenuto fra il muro $\mathrm{O}$ dei castra praetoria e l'agger, là dove si trovava il campus d'esercitazione dei castra ${ }^{34}$ e dove le ricerche archeologiche hanno restituito anche fondazioni di edifici vari, mosaici di ambienti termali, ed altro $^{35}$, relativi all'espansione al di là del più antico limite urbano. Le centinaia di anfore occupavano «un ambiente, con muri in opus latericium spessi oltre $\mathrm{m} 1$, lungo $\mathrm{m} 16$ [...]. L'area era limitata a $\mathrm{S}$ da un muraglione in opus latericium spesso oltre $\mathrm{m} 1$, in direzione EO.». ${ }^{36}$

E gli esempi sono infiniti, poiché buona parte delle città della Gallia Cisalpina ${ }^{37}$ presenta ciascuna un consistente numero di strutture ad anfore. ${ }^{38}$ Fra esse voglio soffermarmi su Mediolanum, perché personalmente a lungo studiata ${ }^{39}$ e tale da poter rispecchiare le peculiarità delle altre antiche urbes padane.

La città è al centro della vasta pianura lombarda, una pianura dalla struttura fondamentale ghiaioso-

M. Antico Gallina. Esse sono frutto delle convinzioni della scrivente e di un confronto con il dott. Geol. Pietro Alemani e con il laureando.

${ }^{33}$ Così come altrove si trovò riempita di calcinacci, scheletri umani e animali, tegole, vasellame vario misti a terra e ciottoli. Fra il tufo vulcanico e le argille dei depositi maremmani doveva collocarsi, pur con variazioni locali, il livello superiore della falda acquifera.

34 Tac. Ann, 12, 36.

35 Steinby 1993: 254. Vd. già la CAR 1977: Tav. III G, nn. 12-19 per le vie Calatafimi e Montebello, prossime all'accumulo anforario in oggetto. Vd. ibid., passim, per ulteriori ritrovamenti lungo la fossa aggeris, come ad esempio il basamento di un'edicola e una grande platea in opus caementicium adiacente ad un accumulo di 103 anfore e 97 colli rinvenuti fra via Montebello e Calatafimi (CAR 1977: tav. III G, n. 6). Non sono noti i rapporti stratigrafici fra tali ritrovamenti.

${ }^{36}$ CAR 1977: tav. IIIG, n. 13-14, a e b. Oltre il muraglione, si rinvenne un ambiente di $\mathrm{m} 4 \times 3$, costruito sui resti di muratura di un vano precedente.

${ }^{37}$ Il moltiplicarsi di questa tecnica nel lasso temporale fra I sec.a.C. e I sec.d.C. va, del resto, di pari passo con il forte incentivo politico all'organizzazione di città secondo quei criteri urbanistici che meglio rispondevano ai caratteri della romanità, in termini di sistema politico, religioso, sociale, amministrativo, economico.

${ }^{38} \mathrm{Vd}$. per uno sguardo di sintesi Antico Gallina 1996a; 1998 a.

${ }^{39}$ Dalla centuriazione, alle strade, ai pozzi, ai ponti, alle peculiarità del suburbium, ai sistemi di bonifica geotecnica ed idraulica, appunto, individuati in ben oltre 60 punti topografici urbani e suburbani: vd. Bibliografia finale di Antico Gallina. 


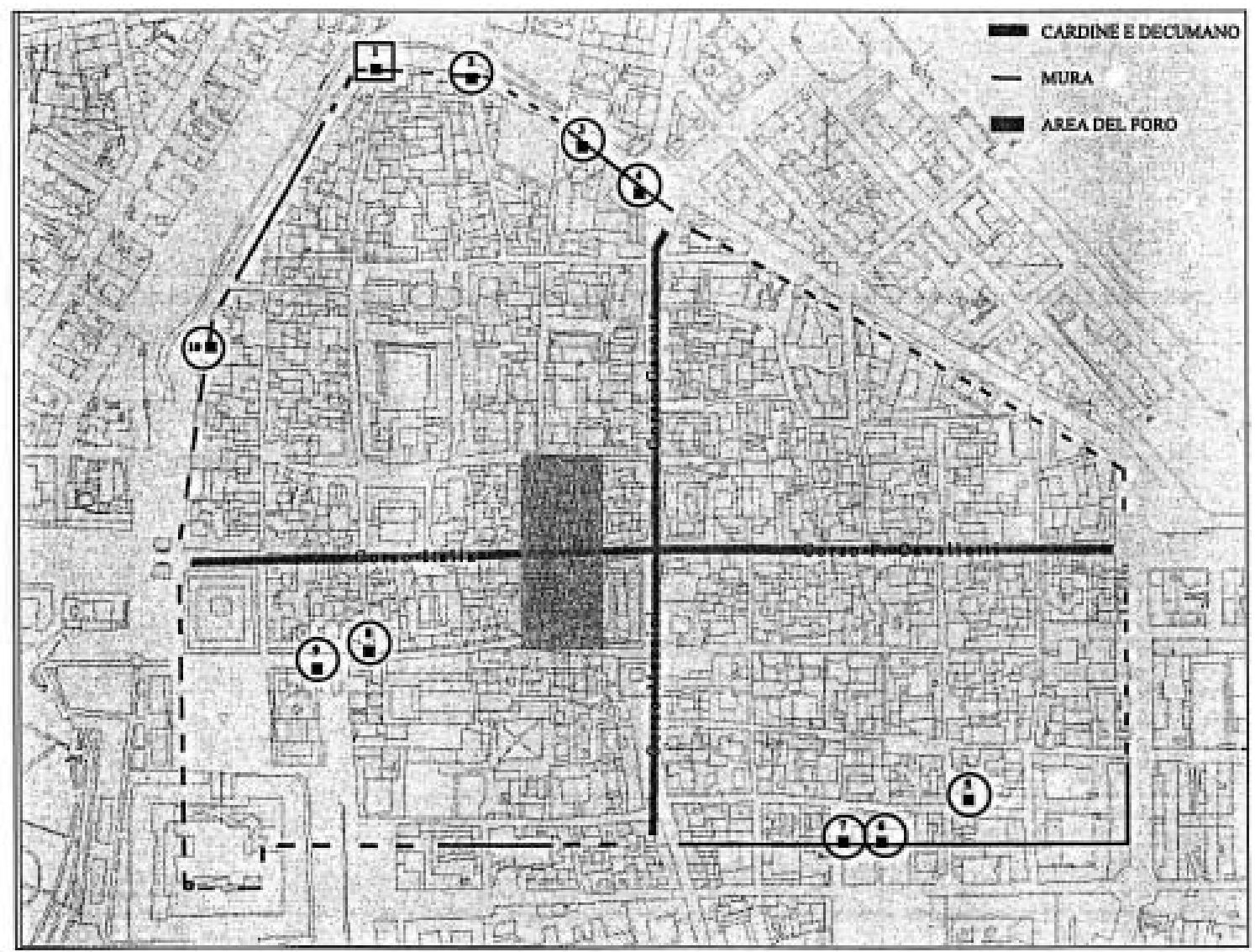

Figura 8. La cinta di Novaria e la posizione degli accumuli anforari nn. 1-10 (da Epifani 2004-05).

sabbiosa, ma è, parallelamente collocata all'interno della fascia longitudinale padana, caratterizzata dal fenomeno della risorgenza della falda acquifera. Argille, ghiaie e sabbie sono incoerenti o quasi, prive di compattezza e vivono nella particolare situazione idrogeologica suaccennata, tale da compromettere ancora di più la loro compattezza: essa ha determinato quella grande variabilità di situazioni litologiche stratificate che vediamo anche nel suolo di $\mathrm{Me}$ diolanum.

Se ad una valutazione macroscopica esso è dunque definibile ghiaioso-sabbioso, ad una valutazione 'microscopica', cioè locale, la variabilità è caratterizzata da una continua alternanza di argille, limo argilloso con alta percentuale di torba.

La fascia padana di terreni saturi risulta tanto più ampia e ricca di punti di risorgenza quanto più procediamo a ritroso nel tempo e giustifica perciò la frequentissima presenza di componenti argillose e torbose. Uno stato di cose che rientra egregiamente nelle condizioni esposte all'inizio di questo saggio e che si presta a soddisfacenti verifiche. I preziosi dati registrati in ambito urbano alla fine del 1800 per degli interventi sulla rete fognaria, permettendo, infatti, il rilevamento delle isopiezometriche, hanno consentito di presumere una non molto dissimile soggiacenza della falda freatica nell'età romana.$^{40}$ La profondità della falda risulta comprovata, del resto, proprio dalla diffusione degli accumuli anforari nel terreno come sistema di bonifica ambientale.

Numerosissimi, dunque, nell' area urbana e suburbana, gli interventi di miglioramento qualitativo del suolo, realizzati in vario modo, sicuramente in considerazione del tipo di utilizzazione cui era destinato il 'soprasuolo', edificio o muro o pavimento o strada $o$ area aperta che fosse. Sostanzialmente le modalità di intervento riconducibili al concetto di bonifica a scopo fondativo sono quelle realizzate con il sistema ad anfore e, parallelamente, con il sistema a pali, a seconda delle esigenze statiche che do-

\footnotetext{
${ }^{40}$ Poggi 1897; Alemani 1996: 17-28 e fig. 10.
} 

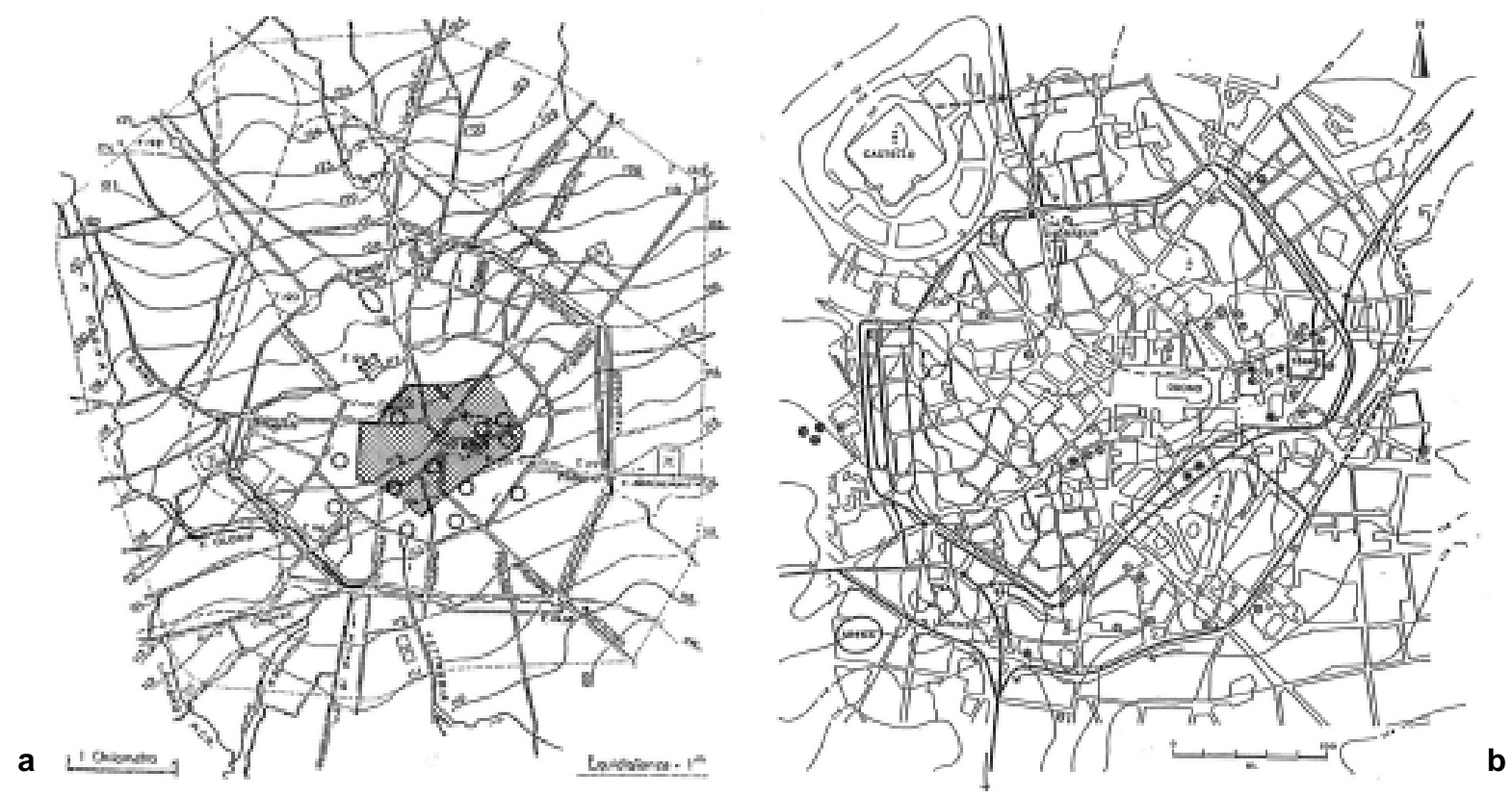

Figura 9 a. Mediolanum, i corsi d'acqua e le isopiezometriche (da Poggi 1897 rielaborata): cerchi = zone urbane e suburbane di maggior concentrazione delle bonifiche; $b$ - città e suburbio in relazione alle isoipse: punto nero $=$ ponti; asterisco $=$ pali di fondazione; cerchio tratteggiato = strutture ad anfore (elaborazione Antico Gallina).

vevano essere affrontate e del 'peso' dell'opera edificata. ${ }^{41}$

A Mediolanum la concentrazione di queste due modalità di fondazioni si presenta, significativamente, nella parte meridionale dell'impianto urbano, dove la granulometria è estremamente variabile. E' la parte bassa della città, considerandone la quota s.l.m., la pendenza e, di conseguenza, la direzione prevalente delle acque superficialiit e di quelle ipogee, nonché la condizione di una falda in età romana affiorante (Fig. 9 a-b).

Non a caso, la situazione descritta coincideva con la maggior dislocazione dei pozzi romani, tutti poco profondi, e, aggiungerei, con il ritrovamento di quattro pompe idrauliche, particolare che, insieme agli altri, mi pare leggibile come una ulteriore riprova non solo di specifiche condizioni della falda acquifera mediolanense, ma anche di sapiente capacità di gestione territoriale. ${ }^{43}$

Date, dunque, le caratteristiche di minimo gradiente piezometrico della falda padana che impediscono il decremento del carico piezometrico se non nel caso

\footnotetext{
${ }^{41} \mathrm{Si}$ pensi al teatro, la cui cavea poggiava su una fitta fondazione a pali. Vd. Antico Gallina 2011b: 125-140.

${ }^{42}$ Verso la città scorrevano tangenti o vi confluivano infatti da settentrione già all'epoca romana fiumi e numerosi torrenti, anche originati da risorgive.

${ }^{43}$ Antico Gallina 1993; 1996 b; 1997 b; 2004a; 2011c.
}

di adozione di un adeguato dreno (lungo canale aperto o tubazione) ${ }^{44}$ 'in nessun caso' le strutture ad anfore rappresentavano dei «drenaggi», motivo per il quale non si possono definire con tale termine. ${ }^{45}$

STRUTture Di Bonifica CON FUNZIONE Di AERAZIONE E DI INFILTRAZIONE

Ci troviamo nell' ambito della bonifica idraulica, all'interno della quale possiamo collocare l' esempio dell'horreum ostiense costruito alla metà del III sec. d. C. presso un' ansa del fiume Tevere. ${ }^{46} \mathrm{Nel}$ piano di anfore poste orizzontali, probabilmente su due ordini sovrapposti, prive di materiali interstiziali e al di sotto di un vano pavimentato, possiamo più facilmente e con maggior certezza riconoscere un'efficace struttura di aerazione capace di intercettare le acque di risalita capillare, grazie ai vuoti dei corpi anforari. Si tratta di contenitori a corpo cilindrico, ${ }^{47}$ di per sé certo meno adatti rispetto alle tipologie maggiormente affusolate a una bonifica di tipo geotecnico con posizione verticale e rovesciata degli stessi. Senza

\footnotetext{
${ }^{44} \mathrm{Vd}$. la fig. 2.

${ }^{45}$ Antico Gallina 1996a: 93-94; 1998a; 2000, 2000c; 2006.

46 Zevi 1972: 404- 431.

47 Tipo Africano grande, Africano piccolo e Lepcis Magna.: Zevi 1972: 416-417.
} 

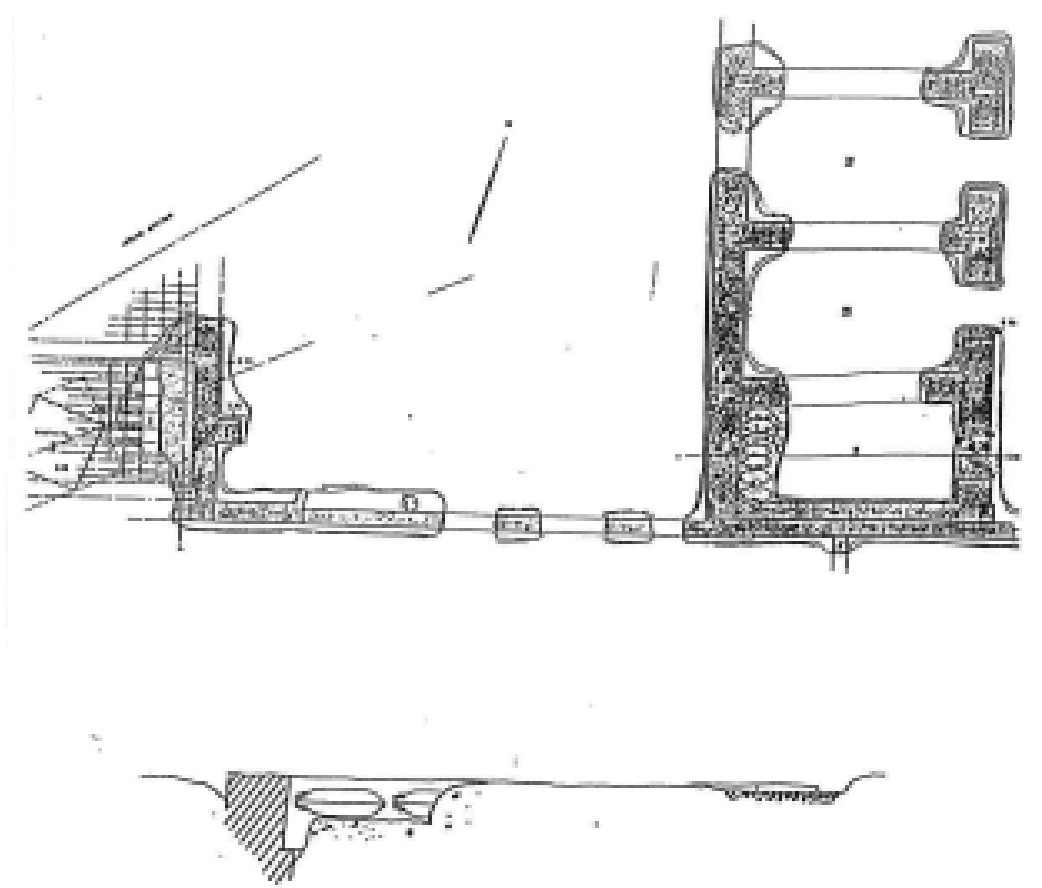

Figura 10. L'horreum ostiense e la struttura di aerazione, pianta e sezione (da Zevi 1972).

considerare che, trovandoci in zona capillare, la sottrazione dell'acqua può avvenire soltanto attraverso i meccanismi di evaporazione. Alla possibile definizione di 'drenaggio permanente' è, per le ragioni suesposte, preferibile usare la definizione di struttura di 'aerazione'.

Un'opera, quella ostiense, atta, dunque, a contrastare l'umidità che, provocando muffe all'interno del locale, avrebbe fatto deteriorare le derrate ivi custodite, ma contemporaneamente a garantire la stabilità termica del vano soprastante, analogamente alla modalità delle suspensurae usate per altri vani dello stesso magazzino ${ }^{48}$ (Fig. 10). L'espansione cilindrica del corpo, comunque in grado di tollerare carichi distribuiti, favoriva quella circolazione d'aria che anche i vuoti lasciati fra le anfore contribuivano ad aumentare.

Possono individuarsi delle condizioni, sempre in zona capillare, che pur potendosi definire con doppio termine, come 'drenaggio temporaneo', è opportuno, onde evitare facili confusioni anche concettuali, indicare con il termine di 'infiltrazione'. Una fossa individuata all'angolo di una domus di Mediolanum pare configurarsi come struttura di infiltrazione o di dispersione delle acque piovane provenienti dal tet-

\footnotetext{
${ }^{48}$ Antico Gallina 1996a: 96.
}

to soprastante per la sua posizione rispetto alla planimetria dell'abitazione, per la sua morfologia (fossa circoscritta e anfore poste su tre ordini), tale da garantire l'aumento della superficie filtrante, per la sua profondità, tale da aumentare la capacità di riempimento, e soprattutto per una valutazione sulla caratterizzazione ambientale. ${ }^{49}$ Le correlazioni stabilite fanno ritenere che la zona capillare potesse avere momentanee condizioni di saturazione legare ad eventi meteorici od a carente regimazione delle acque superficiali.

\section{StRUtTURE DI DRENAGGIO}

Sul 'mondo del drenaggio' mi pare ci si sia soffermati a sufficienza. In zona satura, ove costantemente domina l'acqua gravifica che occupa tutta la porosità disponibile e che si muove prevalentemente in direzione orizzontale, l'opera drenante presuppone uno scavo che con la depressione della superficie piezometrica induca il movimento dell'acqua verso l'opera drenante. Ma l'opera deve prevedere una via

49 Antico Gallina 1996a: 93. Pur permanendo dubbi, in quanto i dati pubblicati non risultano mai esaustivi. Non sappiamo, nel caso di questo ritrovamento, se ci fosse materiale interstiziale o no, e, nel caso, in che cosa consistesse. 
di deflusso delle acque raccolte, tale da consentire un decremento del carico piezometrico. Dalla letteratura dedicata ai sistemi ad anfore non riscontro presenti dati tali da far ricondurre la struttura alla funzione di drenaggio (quote inizio e fine accumulo, sua lunghezza totale, pendenza terreno, gradiente piezometrico). Anche solo il fatto che la lunghezza totale non sia mai riscontrabile con certezza rende la lettura della 'funzione drenante' una pura ipotesi.

Un caso occorso nell'antica Lodi (Laus Pompeia) potrebbe dare l'idea di una bonifica idraulica con funzione di drenaggio, con la quale gli antichi attuarono un 'drenaggio' vero e proprio in quella precisa zona extraurbana. Le anfore, incastrate fra loro, in tre file parallele e sovrapposte su tre ordini, ${ }^{50}$ potrebbero a buon diritto essere una parte o l'inizio di un canale qualora si potesse, però, riscontrare soddisfatto il necessario rapporto lunghezza struttura-gradiente piezometrico. $^{51}$

Altro possibile esempio di drenaggio vero e proprio mi pare quello di Lione, La Solitude, sulla collina di Fourvière: ${ }^{52}$ due livelli di anfore Dr. 20 poste lungo un muro potevano fungere egregiamente da dreno delle acque 'collinari', qualora però fra il muro e l'accumulo e fra gli interstizi si trovasse del materiale permeabile, dato non riportato.

Altra cosa, invece, è la condizione di una fila di anfore o di corpi anforari incastrati, spesso definita «drenaggio», mentre si tratta piuttosto e più semplicemente di canalizzazione/ condotto. ${ }^{53}$

\footnotetext{
${ }^{50}$ Jorio, Donati et alii 2005: 20.

${ }^{51}$ Per la stessa ragione si pone in forma dubitativa la serie di accumuli lineari e ravvicinati e quelli a fossa individuati sempre a Lodi Vecchio, via don Milani (Ridolfi 2006: 94-95) non distanti dai precedenti(vd. nota 50), considerati «drenaggi». Quando la verifica del rapporto lunghezza dreno- acclività- gradiente piezometrico non corrisponde alla reale situazione del ritrovamento (vuoi perché l'accumulo è stato, nel tempo, in parte distrutto, vuoi perché giuntoci integro, ma di lunghezza non idonea a drenare), si deve rinunciare al termine 'drenaggio' e ricercare nell'accumulo altre più oggettive funzioni. Il terreno, infatti, per quanto di scheletro ghiaioso presenta lenti limose e argillose e la vasta zona fu interessata dalle condizioni di quello che oggi è il Cavo Sillaro, ma che ebbe una pregressa storia di 'fiume' di risorgiva con un suo alveo ben definito, come è ben evidente dalle fotografie aeree e dalla cartografia gelogica, oltre che dallo stesso tessuto topografico. Ciò può condurre verso l'esigenza di opere geotecniche oppure idrauliche, come quelle con scopo di infiltrazione delle acque superficiali di ristagno riscontrabili in una delle necropoli di Oderzo: Antico Gallina1996a: 96 (necropoli di via Spinè).

${ }^{52}$ Bruhl 1964: 413, riportato anche da Laubenheimer 1998: 58 («mur protégé des eaux... «).

${ }^{53}$ Penso a Bibracte ed alla condotta di corpi di anfore Dr. 1 (Laubenheimer 1998: 48), la cui funzione di «drenaggio» sarebbe giustificata solo da uno sviluppo in lunghezza relazionato alle quote ed alla pendenza del terreno.
}

\section{ALCUNI CASI IN HISPANIA}

Stante quanto fin qui illustrato, fra i ritrovamenti ispanici, la documentazione di Saragozza mi è parsa fra quelle che maggiormente si adattano al confronto con quella cisalpina. La città è situata nella conca idrografica di massimo potenziale d'inondazione corrispondente al bacino del fiume Ebro, a ridosso di esso e vicino alla confluenza del rio Huerva nell'Ebro. ${ }^{54}$

Nello scavare (1928) il terreno fra la cinta tardoromana in parte inglobata nel perimetrale del convento del S. Sepolcro e quella che lo separa dalla «Ronda del Ebro», oltre a molta ceramica spiccava un deposito d'anfore di diverse dimensioni: ${ }^{55}$ sotto lo zoccolo di fondazione della cinta l'architetto La Figuera individuò uno strato di argilla alluvionale spesso cm 40 e subito sotto l'accumulo di anfore. Databili alla metà del I sec. d. C., furono correlate alla fondazione di una prima cinta distrutta da quel fenomeno esondativo ${ }^{56}$ cui, secondo La Figuera, si doveva il deposito argilloso, ${ }^{57}$ e lasciate in situ all'atto della realizzazione della cinta tardoimperiale.

Nel 1935 un altro, non distante, esteso giacimento di anfore infisse ordinatamente e capovolte, vicino al Ponte del Pilar, fece supporre ci si trovasse di fronte a elementi pertinenti ad un porto fluviale. ${ }^{58}$ Considerato anche come uno stoccaggio di contenitori in attesa di imbarco, prova dei traffici commerciali di Caesaraugusta attraverso Ebro e, forse, Huerva, ${ }^{59}$ il tipo di ritrovamento si presta ad una puntualizzazione.

Ora, le anfore, individuate alla profondità di $\mathrm{m} 3$ (perciò a q. m 196 s.l.m.), ${ }^{60}$ erano collocate con molta diligenza entro uno strato arenoso dello spessore di ca. $\mathrm{m} \mathrm{1,} \mathrm{ed} \mathrm{inoltre} \mathrm{la} \mathrm{zona,} \mathrm{oggi} \mathrm{interna} \mathrm{a} \mathrm{Saragoz-}$ za, fra calle de Alonso V e il barrio de Las Tenerías, corrispondeva in età romana a quel suburbium che vide il sorgere di ricche ville ${ }^{61} \mathrm{e}$ fattorie, sicuramente

\footnotetext{
${ }^{54}$ Beltàn 1961: 67 menziona proprio la zona bassa e inondabile di Saragozza

55 Beltràn Lloris 1991: 108.

${ }^{56}$ Poco convincente l' ipotesi che la potenza esondativa sia stata tale da abbattere una cinta muraria.

${ }^{57}$ Solo però l'analisi dello strato d'argilla spesso $\mathrm{cm} 40$ si tratta di un deposito 'turbato', legato alle correnti d'acqua? Ha trascinato con sé dei materiali? - avrebbe potuto dare certezze sulla sua natura alluvionale.

${ }^{58}$ Lostal Pros 1978: 90, con rimando bibliografico.

59 Beltrán 1951: 497-506; Id. 1961: 74, ricorda 1'antico nome Orba del rio Huerva e l'esistenza della città di Botorrita lungo la sua valle.

${ }^{60}$ Pare essere la stessa quota del pelo della falda antica, se nel contesto della non distante villa romana il pozzo verso la vicina calle Rebolerìa aveva una profondità di $\mathrm{m} 3$ (Beltrán 1951: 498-499).

${ }^{61}$ Come la villa individuata fra la calle de Alonso V e Rebolerìa: cfr. Lostal Pros 1978: 99-100, con rimando bibliografico.
} 
collegabili ai traffici fluviali, ma contestualmente non scollegabili da un dato ambientale altamente significativo: la parte di suburbio prescelta per l'espansione corrispondeva alla zona più bassa della città, a margine del rio Huerva ${ }^{62}$ e per giunta nel settore della sua confluenza nell'Ebro e dunque con un'inclinazione del piano urbano verso il letto del rio.

Al riguardo Beltrán Lloris nel 1977 modificò la prima ipotesi di uno stoccaggio, in quella di un'opera per drenare il terreno ed alzarne il livello, realizzata fra il 14 e il 51 d. C. ${ }^{63}$

Il più recente recupero risale al 2004, proprio nella piazza di Las Tenerías ${ }^{64}$. Alla quota di m 194,051 s.l.m. un accumulo di ben 814 anfore è considerato relativo alla realizzazione, in età augustea, di un ampio piano, a fondamento di muri di contenimento. Concordo con gli Autori sulla esigenza di risanamento del terreno a ridosso del rio Ebro, risanamento che io considererei di tipo geotecnico. La struttura inoltre, proprio perché costituita da anfore vuote e coperte di ghiaia, assolse il compito di struttura di aerazione. Riterrei quindi più consona, in luogo del termine «drenaggio» adottato, parlare di aerazione perché opera in ambiente imbibito, atta a contrastare la risalita dell'umidità capillare, e non ad allontanare l'elemento liquido. Del resto, il sovrapposto livello di argilla depurata, buon elemento «sigillante» se in condizione di ambiente asciutto, può -a questo punto- aver assolto questa sua funzione proprio grazie alla struttura di evaporazione sottostante (anfore + ghiaia). Proponiamo tale ritocco interpretativo alla luce delle esperienze di cui si è in precedenza parlato.

In verità, una prima osservazione fattibile è che nella zona dalla q. di m 203 s.l.m. si passa rapidamente alla q. di m 199 (calle del Coso) e che l'andamento delle quote di m 199 e 198 s.l.m. rimarca un'apertura depressiva suscettibile di fenomeni esondativi o di ristagno. Le caratteristiche topografiche e ambientali (quote, pendenza e direzione dei corpi idrici, grado di imbibizione del terreno), ritengo siano proprio quelle che devono aver suggerito non tanto un'opera di drenaggio (poco realistica rispetto alla persistenza delle condizioni ambientali come la falda subaffiorante), quanto un più pratico ed efficace metodo di bonifica geotecnica che restituisse consi-

\footnotetext{
${ }^{62}$ Dal Campo di Santa Engracia all'imbarcadero.

${ }^{63}$ Beltrán Lloris 1977: 392, 395 e nota 848. Le anfore appartenevano ai tipi Dr. 7, 7/8, 10, 12, 22, 1, 24.

64 Cebolla Berlanga, Domínguez Arranz, Ruiz Ruiz 2004:464- 467. Per quelli che vengono definiti i sistema di drenaggio di Saragozza vd. anche Domínguez Arranz, Vallejo Rasero, Ruíz Llera 2003: 85.
}

stenza ad un terreno assai cedevole, ma da utilizzare, ${ }^{65}$ e riqualificasse un settore periurbano per la sua natura problematico, ma per la politica economica della città in decisa espansione.

Saragozza oltre che essere collocata nella piana alluvionale di un grande fiume, ha terreni caratterizzati da ghiaie, sabbie e limo, con presenza di argille e con un livello di falda superficiale, specie nell' area della confluenza del rio Huerva nell'Ebro. ${ }^{66}$ L'ipotesi che tale confluenza del rio potesse aver formato un'insenatura interessata da un imbarcadero è riportata da Antonio Beltràn, unitamente alla memoria del «deposito d'anfore». ${ }^{67}$

Ma un altro (1996) caso ispanico vale la pena di citare, quello dell' accumulo rinvenuto a Cadice, località Los Cargaderos, nella zona sud di S. Fernando, laddove l'ambiente costiero pur presentando la colmatazione dei condoni costieri che circondavano l'isola di S. Fernando dalla protostoria, dovuta agli apporti sedimentari trascinati da Rio Guadalete e da altri fiumi secondari, manteneva terreni imbibiti e aree paludose (Fig. 11).

Compiutamente descritto nelle peculiarità formali e nella stratigrafia litologica, ${ }^{68}$ l'opera presentava uno strato $(\mathrm{cm} \mathrm{15)}$ di ghiaia e ciottoli di piccole e medie dimensioni mescolati al «fango, di tessitura sabbiosa» e colore marrone molto scuro, con frammenti ceramici; esso copriva 2 «sublivelli», A e B, dalle seguenti caratteristiche:

A: simile al livello superiore, ma con ghiaia scesa di quota e mescolata con fango marrone -grigiastro del terreno paludoso. Era questo lo strato a contatto con i livelli di deposizione delle anfore ed entro le file da esse formate;

B: strato compatto di fango grigio caratteristico del terreno paludoso in cui era la struttura ritrovata, spesso ca. $\mathrm{m} 1$.

Le caratteristiche dell'accumulo erano:

1: fila di anfore (conservati 2 allineamenti) poste coricate, molte frammentarie, con puntale infilato nella bocca dell' anfora successiva. In direzione opposta una seconda fila di anfore orizzontali;

2: altre 2 file di anfore complete, poste coricate. Vari pali di pino appuntiti situati tra le file di anfore, posti in verticale nel fango. Servivano da conte-

\footnotetext{
${ }^{65}$ Forse come riempimento e consolidamento del terreno in vista del 'peso' della prima cinta, conservato poi al tempo della costruzione della cinta di III secolo? La verifica delle sezioni di scavo, delle quote e la valutazione delle peculiarità fisico-meccaniche del suolo direttamente interessato dalle mura potrebbero fornire qualche chiarimento.

${ }^{66}$ Mapa geològico, 1994, terreni tipologia 101 e 102.

${ }^{67}$ Beltrán 1961: 73 e nota 19, con rimandi bibliografici..

${ }^{68}$ Bernal, Sàez et alii 2005: 185-194.
} 


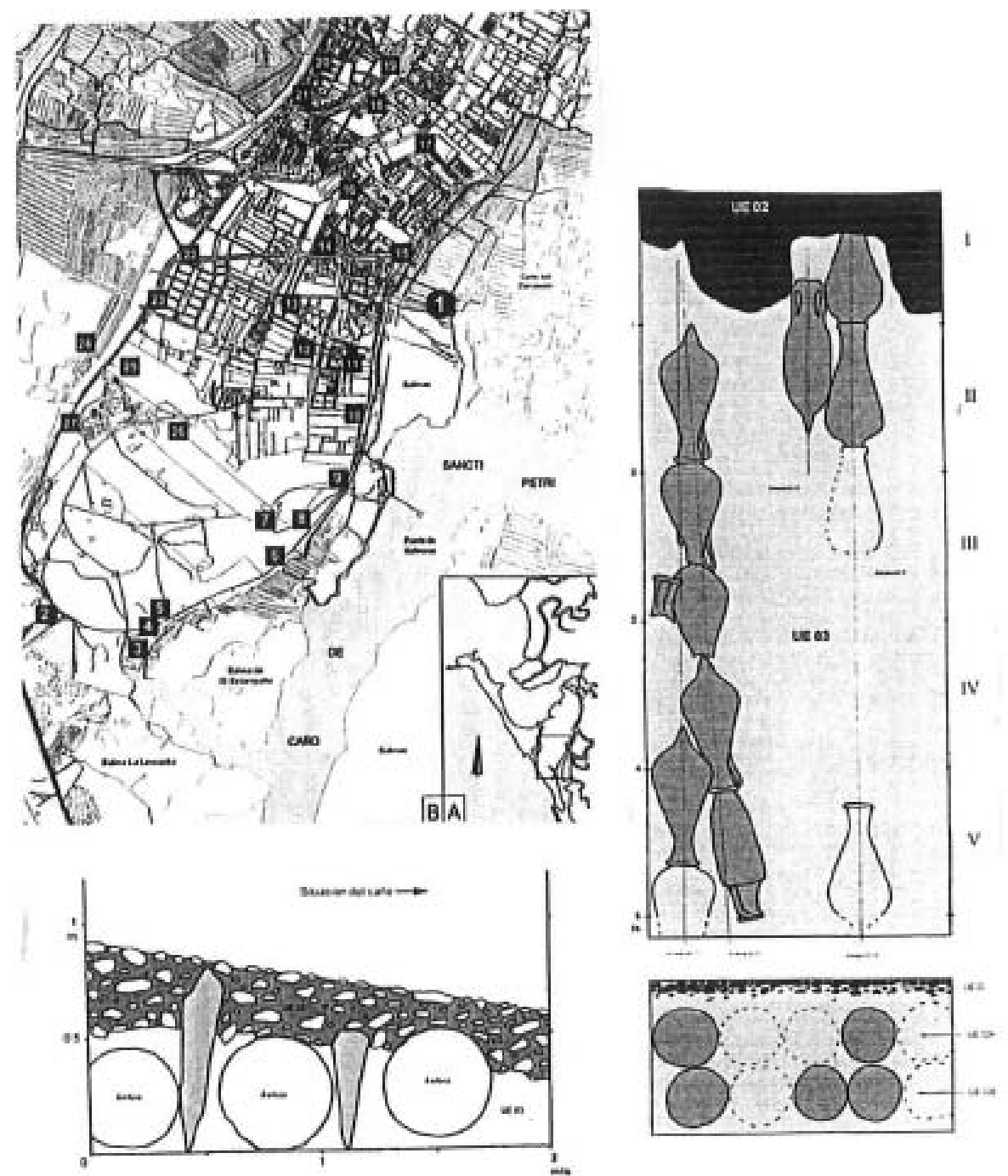

Figura 11. Cadiz, località Los Cargaderos. Le caratteristiche della struttura ad anfore in pianta ed in sezione ( da Bernal et alii 2005).

nimento delle file di anfore per impedire lo spostamento orizzontale; uno di essi aveva 4 fori longitudinali (diam. cm 1,8/2,5), la traccia di una corda o di un piccolo pezzo di incasso sempre di legno.

Estrapolando, ora, dalla Scheda-tipo complessa elaborata e proposta da chi scrive per il censimento degli accumuli anforari, ${ }^{69}$ alcune parti e semplificandone l'uso, ${ }^{70}$ potremmo vedere di collocarvi i dati

\footnotetext{
${ }^{69}$ Antico Gallina 2008b: 22-33; 2011a.

${ }^{70}$ La Scheda originale, per la quale vd. nota 69 , nella sua qualità di Scheda -tipo destinata ad essere utile in ogni situazione documentaria e, come tale, immodificabile, prevedeva oltre alla voce Struttura unitaria anche quella di Struttura articolata, atta a contemplare i dati relativi ad eventuali restau-
}

riportati dalla bibliografia e quelli parzialmente interpretati da chi scrive per porre in maggior rilievo gli elementi utili all'interpretazione funzionale. La porzione di Scheda sintetica (Fig. 4) che fa seguito riporta, rispetto a quella complessa, due sole Aree d'informazione, quella Descrittiva e quella Tecnica, relativa cioè al Modello stratigrafico. Quest'ultimo segue il grafico teorico (Fig. 3) che ne evidenzia le sezioni longitudinali e trasversali utili all'interpretazione delle funzioni:

ri o modifiche degli accumuli. Per facilità di lettura in questa sede si sono ulteriormente ridotte le voci, al fine di concentrare l'attenzione sull'accumulo di Cadice. 
AREA DESCRITTIVA

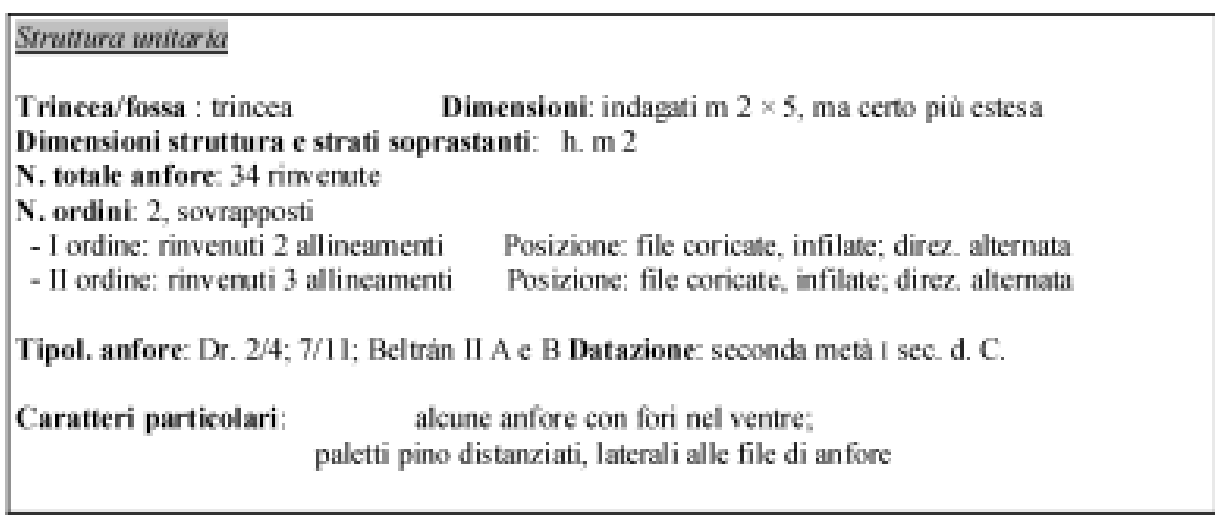

\section{AREA TECNICA \\ MODELLO STRATIGRAFICO (fig. 9)}

Sintesi litologia/materiale in relazione con la struttura ad anfore

\begin{tabular}{|c|c|c|c|c|}
\hline & SOPRASTANTE & SOTTOSTANTE & LATERALE & INTERSTIZIALE \\
\hline Litologia & $\begin{array}{l}\text { 1-cm } 15 \text { di ghiaia, } \\
\text { ciottoli di piecole } \\
\text { e medie } \\
\text { dimensioni con } \\
\text { “ango, di } \\
\text { tessitura sabbiosa" } \\
\text { e di colore } \\
\text { marrone molto } \\
\text { scuro } \\
2 \text {, a contatto con } \\
\text { struttura- simile } \\
\text { al livello 1, ma } \\
\text { con ghiaia scesa } \\
\text { di quota e } \\
\text { mescolata con } \\
\text { "fango marrone - } \\
\text { grigiastro" }\end{array}$ & $\begin{array}{l}\text { materiali limosi } \\
\text { grigi, sterili }\end{array}$ & $\begin{array}{l}\text { materiali limosi } \\
\text { grigi }\end{array}$ & $\begin{array}{l}\text { I ordine;ghiaia } \\
\text { dello strato } \\
\text { soprastante, scesa } \\
\text { di quota e } \\
\text { mescolata a } \\
\text { "fango marrone - } \\
\text { grigiastro"; } \\
\text { II ordine; limo } \\
\text { compatto grigio }\end{array}$ \\
\hline Materiale & $\begin{array}{l}\text { 1-Frr, ceramici, } \\
\text { soprattutto anfore, } \\
\text { di piccole } \\
\text { dimensioni } \\
\text { mescolati al } \\
\text { "fango" }\end{array}$ & & $\begin{array}{l}\text { paletti di legno di } \\
\text { pino }(\mathrm{cm} 75,5 \times \\
18,5 \times 9) \text { con fori. } \\
\text { tra le file di anfore }\end{array}$ & \\
\hline
\end{tabular}

Sono a mio parere prospettabili alcune osservazioni. Il piano di battuto $(\mathrm{cm} \mathrm{15)}$ pare costituisse il livello più superficiale dell'intera opera fondazionale e sembra atto ad uniformare il basamento, onde evitarne un cedimento differenziale. Il colore marrone molto scuro dell'elemento coesivo rimanda a condizioni deposizionali in fase di colmatazione terminale con paludi torbose, cui è stato dall'uomo aggiunto il materiale più consistente formato da ciottoli, ghiaie e frantumi ceramici. Il livello basale di questo strato è in parte confluito nel tipo di materiale sciol- to in cui fu immesso l'accumulo anforario. Il colore marrone-grigiastro del terreno a diretto contatto con la struttura ad anfore e il grigio del sedimento sottostante e laterale alla struttura segnala quell'ambiente caratterizzato da acque asfittiche, riducenti e stagnanti quali paludi e stagni, appunto, che vanno a aumentare la sostanza organica con conseguente formazione di torbe e rimarca, a mio parere, la condizione di un settore con una capacità di portanza nulla o molto bassa. Un settore, dunque, che in un progetto di riconversione in banchina $\mathrm{o}$ in 
camminamento doveva necessariamente essere bonificato.

Le analogie che riscontro con le spianate anforarie di Cremona, di Padova, di Parma, ma anche di Massalia, mi inducono ad interpretare il manufatto, che gli autori della notizia considerano più esteso dal momento che i pali non ne indicavano la terminazione, come un'opera di consolidamento, una munitio di un suolo molle (lagunare), cedevole rispetto alle esigenze di 'carico' al quale lo si voleva sottoporre. I paletti lignei legati fra loro potrebbero aver assolto il compito di rinforzare lateralmente l'intera struttura, per contenere gli eventuali spostamenti e preparare, costipandolo, il terreno all'incremento di carico del corpo costituente il camminamento e quindi essere usati quali elementi di fondazione di un camminamento/argine/molo (?), come peraltro è tipico nei terreni paludosi; ${ }^{71}$ la presenza di fori, sebbene non su tutte le anfore, può far pensare, più che alla funzione di 'antigalleggiamento' applicabile nei casi padani con falda freatica subaffiorante e numerosi fori, ${ }^{72}$ alla creazione di un manufatto stabile, ma flessibile: con l'alta marea esso poteva subire oscillazioni, ma non distruggersi, anche perché rinforzato dalla palificata di contenimento. Poteva, così, comunque garantire la percorribilità della strada/alzaia e, forse, contestualmente fungere da opera di contenimento spondale ${ }^{73}$ del Caño de San Pedro, in un'area connotata da una rete di canali. ${ }^{74}$ Posso concordare con la definizione di «pequeño dique» adottata dagli studiosi spagnoli, che intenderei con il significato di elemento di contenimento, mentre non collima con il mio pensiero il «pequeño dique, realizado con un sistema de drenaje utilizando ánforas». ${ }^{75} \mathrm{~A}$ mio avviso anche per questo accumulo il concetto, come la definizione, di drenaggio risultano contrari non solo alle peculiari-

\footnotetext{
${ }^{71}$ Viene alla mente la varia metodica usata per la realizzazione delle strade in ambiente paludoso (pontes longi) e conosciuta già dai tempi preistorici.

${ }^{72}$ Antico Gallina 1996a: 85. Fori e rotture intenzionali sulle anfore avrebbero potuto, se presenti sulla totalità o sulla maggioranza dei contenitori ( come in alcuni accumuli rinvenuti nell'area dell'Università Cattolica di Milano, considerati in Bernal, Sáez et alii, 2005: 193 come un sistema di più rapida evacuazione dell'acqua pluvia), ben contrastare quella piccola risalita d'acqua ipogea che avrebbe generato leggere spinte verso l'alto: la fuoriuscita dell'aria dai fori avrebbe consentito all' anfora il riempimento di acqua con conseguente stabilizzazione dell'accumulo. Una serie di fori o rotture equivalenti ( ad esempio la mancanza del puntale) in accumuli che presentano materiali interstiziali non permeabili, poteva rendere le anfore usabili alla stregua di veri tubi di aerazione.

${ }^{73}$ Bernal, Sáez et alii 2005: 214.

${ }^{74}$ Bernal, Sáez et alii 2005: 216.

${ }^{75}$ Ibid.: 213.
}

tà ambientali, ma alla funzione riconoscibile, quale manufatto per la creazione di un camminamento o di una banchina, atta a sostenere carichi, come, ad esempio, il passaggio di carri, a facilitare dunque, come dicono giustamente gli Autori, l'accesso al canale. ${ }^{76}$

Come già si è detto, nel mio linguaggio, mutuato da quello dei geologi, il termine drenaggio viene riservato ad opera che capta acqua e la allontana; nella zona paludosa del Caño de San Pedro drenare voleva dire portare via acqua con una struttura molto, ma molto lunga.

Nei casi di ambiente roccioso o di terreni cementati la presenza di strutture ad anfore a maggior ragione comporta una descrizione stratigrafica rigorosa e esaustiva, seppur sintetica, mancando la quale ci si deve limitare a ipotesi anche duplici.

Il caso di fosse, talora in origine di uso sacrale, convertite in pozzi neri o immondezzai e colmate poi, in funzione dell'uso del suolo per nuove attività edilizie, non ci pone di fronte ai classici interventi di bonifica di terreni problematici, ma di fronte a una più efficace modalità per ovviare alla difformità creatasi nel piano fondale. Questo, sebbene anche tale tipo di intervento sia inquadrabile all'interno della metodica geotecnica, in quanto il materiale scelto per il riempimento era in grado di costipare il settore di suolo incavato (fosse) evitando quei cedimenti superficiali o quelle depressioni che si sarebbero verificate qualora fosse stato privilegiato materiale naturale, argilloso/ sabbioso e quindi migliorando le sue capacità di portanza, nell'eventualità di sovrapposizioni di opere edilizie. L'area del foro di Ampurias per la cui costruzione si procedette a colmare avvallamenti e irregolarità del sostrato roccioso, di natura calcarea, può essere un esempio dell'attività sopraindicata, una tipologia di scelta della quale erano evidentemente molto chiari agli antichi costruttori i vantaggi correlati.

Riempimenti con terra e materiale ceramico fra cui frammenti anforari tardo-repubblicani finalizzati a livellare tali difformità della roccia-base calcarea sono stati individuati in vari settori dell'area occupata dal foro, ${ }^{77}$ ad esempio lungo l'ambulacro ovest, ma anche nel settore est del criptoportico al di sotto del muro N/S che delimitava a est il criptoportico: qui due depositi a fossa, a ca. 2 metri di distanza fra loro (denominati Gall 1 e Gall 2) risultano l'esito di un intervento sincronico, perché riempiti di materiali frammentari omogenei, provenienti

\footnotetext{
${ }^{76}$ Ibidem.

${ }^{77}$ AA.VV. 1984: 23, 36, 368. Per il foro repubblicano di Ampurias vd. anche Ruiz de Arbulo Bayona 2001: 27-29.
} 
da un'unica discarica. ${ }^{78}$ La sequenza stratigrafica individuata già nel settore delle cisterne ${ }^{79}$ è confrontabile con l'esito di altri saggi, anche ad di fuori dell' area forense, quali indizi delle opere di adeguamento fondazionale che hanno preceduto sia l'impianto della città sviluppatasi dal praesidium militare romano e del foro repubblicano, sia la sua ristrutturazione in età augustea. ${ }^{80}$

Un buon parallelo a tale condizione ambientale è individuabile in ambito provinciale insulare: a Malta. All'interno del municipium di Melita alcuni accumuli con funzione di circoscritto apparato fondazionale in corrispondenza di rari punti all'interno di domus troverebbero giustificazione quale risoluzione idraulica a fessurazioni o cavità della roccia calcarea su cui furono costruite le dimore, cavità riempite dal colluvio di materiali organici e terrosi. ${ }^{81} \mathrm{I}$ modesti accumuli maltesi fanno pensare più che a interventi geotecnici a modalità idrauliche atte a risanare pavimento e ambienti soprastanti.

\section{CONSIDERAZIONI DI METODO E DIREZIONI DI SVILUPPO}

\section{LE ESIGENZE DEL TERRENO}

Il concetto che supporta l'analisi degli accumuli sta, per chi scrive, nel fatto che, valutate litologia, pendenza del territorio, gradiente piezometrico e spessore della falda freatica, non potendo drenarla, né essendo noti casi di ritrovamenti di condotti drenanti di lunghezza adeguata, ${ }^{82}$ i tecnici dell'antichi-

\footnotetext{
${ }^{78}$ Ibid.: 369: nella fossa Gall 1 di 758 frammenti di anfore italiche,il $35,75 \%$ appartiene al gruppo di produzione pompeiana o comunque campana; in quella Gall 2 di 372 frammenti il 31,84\% appartiene allo stesso gruppo. (ultimo quarto II sec.a.C.- primo quarto I sec.a.C.). Erano presenti anche frammenti di anfore puniche assimilabili alla forma Mañà $\mathrm{E}$.

${ }^{79}$ Ibid., 427-463.

${ }^{80}$ Pongo in forma dubitativa il confronto con Tarraco. La persistenza di alcune delle discariche di frantume ceramico e anforario (V sec. d.C.)individuate sulla parte elevata, sede del cosiddetto foro provinciale, nelle fasi medievali di ricostruzione (pare il caso dell'Hospital Vell de Santa Tecla, XII secolo), fa considerare, al di là della funzione di «ottenere il livello di circolazione dell'area» suggerita per calle Villaroma (Ted'A 1989:171), il fatto che nel momento di riconversione degli spazi in aree abitative, la situazione di tali riempimenti, per quanto assimilabile a quella dei terreni di riporto e quindi passibile delle stesse problematiche e carenze, potesse risultare efficace quale opera di protezione dall'umidità dovuta a infiltrazione od a scorrimento, o a risalita per capillarità. Le risposte, anche in tale caso, stanno nell' interazione terreno (conglomerato)-discarica. Per il foro tarragonese rimando anche ai numerosi saggi contenuti in Mar 1993.

${ }^{81}$ Antico Gallina 2004b: 245-271.

${ }^{82}$ Cfr. sul tema Antico Gallina 1996a; 1998a.
}

tà operavano secondo l'unica soluzione possibile: quella di opporsi allo stato del terreno e renderlo resistente non certo asciugandolo, ma costipandolo, con anfore o con pali. Il loro 'lavoro' all'interno del terreno è, del resto, chiaro, ${ }^{83}$ tale da rendere fattibile l'edificabilità di opere anche d'impegno.

In generale, le strutture ad anfore appaiono poste entro trincee od in fosse quadrangolari o circolari, lungo corsi d'acqua, al di sotto di vie di comunicazione, a fianco od al di sotto di muri, di aree aperte (cortili, spazi liberi fra abitazioni, aree a diversa destinazione), di pavimenti nell'edilizia prevalentemente privata, con evidente funzione sia di bonifica geotecnica (di fondazione, di consolidamento del terreno, di 'sostegno' del terreno, ${ }^{84}$ di stabilizzazione dell'opera sovrapposta) che idraulica (sistemi di aerazione ${ }^{85}$ talora di infiltrazione, di isolamento o di stabilizzazione termica, ${ }^{86}$ di aumento del deflusso idrico verso strutture di approvvigionamento, ${ }^{87} \mathrm{di}$ antigalleggiamento ${ }^{88}$ ).

Non infrequente l'uso di strutture ad anfore quale opera di contenimento spondale, in caso di rive dalla composizione fine, limosa, quindi cedevoli, o di difesa spondale nel caso, invece, di un fiume che vada ad erodere la riva.

In definitiva, l'approccio interdisciplinare — che vede la necessità di sinergie fra archeologi e geologi nella lettura dei terreni durante le fasi di scavo e nella verifica del dato interpretativo ${ }^{89}$ - , approccio ribadito da chi scrive ${ }^{90}$ anche in occasione del Convegno sul tema «Bonifiche e drenaggi con anfore in epoca romana», ${ }^{91}$ è stato del resto avvalorato dagli studi sempre più numerosi, proporzionali ai sempre più frequenti ritrovamenti di accumuli anforari. La mia posizione metodologica è quella di ribadire l'essenzialità di un rigoroso riconoscimento delle peculiarità stratigrafiche a contatto con gli accumuli (Fig. 3), descritte in base agli standard

${ }^{83}$ Antico Gallina 1996a: 94-95; Ead. 1998b: 91-109.

${ }^{84}$ Ritengo di poter individuare tale funzione nei casi dei noti puits funéraires, che si commentano in Antico Gallina 1996a, 101-103 e Ead. 1998a, pp. 76-77.

${ }^{85}$ Come il caso dell'horreum ostiese presso la riva del Tevere, per il quale vd. supra.

${ }^{86}$ Vd. per Augusta Praetoria (Aosta) Antico Gallina 2008a.

${ }^{87}$ In relazione ad un pozzo individuato a Mediolanum e caratterizzato da una fascia basale formata da 5 file sovrapposte di colli d'anfora in parte con puntale a mo' di tappo,per richiamare 1'acqua e per filtrarla (Antico Gallina 1997a: 143). ${ }^{88}$ Antico Gallina 1996a: 85.

${ }^{89}$ Rimando in tal senso ad Antico Gallina 1996a e 2008b.

${ }^{90} \mathrm{E}$ usualmente applicato in studi relativi alla gestione dei territori, a partire da Antico Gallina 1982.

${ }^{91}$ Cfr. Antico Gallina 1998a: 73-79, intervento a segnalazione degli esiti dello studio Ead. 1996a, allora già in stampa. 
internazionali di riferimento per la geotecnica. ${ }^{92}$ Potrebbero, in questo modo, aprirsi ulteriori, forse insospettati, orizzonti interpretativi, utili alle conoscenze dell'ambiente nell'antichità e di altri suoi possibili problemi, come ad esempio i numerosi fattori di inquinamento, nonché della 'sensibilità' tecnica dei costruttori.

\section{LA TIPOLOGIA ANFORARIA}

Si è ampiamente riscontrato come i costruttori privilegiassero per la realizzazione di opere di bonifica, contenitori anforari con corpi allungati e con fittone pronunciato: la forma $\operatorname{Dr} 6$, soprattutto nella variante b, è in assoluto quella prevalente, ma buona è l'attestazione di anfore Dr. 7/11, di produzione ispanica, anch'esse di corpo oblungo. ${ }^{93}$ Leggere, perché vuote, dal peso specifico molto basso, ma con pareti spesse dalla elevata microvacuolarità dell'impasto, peduncolo pieno e una resistenza ai carichi elevatissima, ${ }^{94}$ le anfore erano in grado di trasmette al terreno sottostante il carico distribuito, con un lavoro correlato al tipo di terreno con cui erano a contatto (lavoro di punta o di attrito laterale) analogo alle più consuete strutture di fondazione/ sottofondazione in suoli problematici, quelle a pali. ${ }^{95}$ Nella casistica che abbiamo avuto modo di considerare la discriminante fra uso del sistema ad anfore ed uso del sistema a pali sta nella prevalenza del secondo per edificazioni di maggior impegno architettonico (edilizia pubblica, infrastrutture).

Al di là delle variabili nell'andamento del mercato, la tipologia anforaria può rappresentare un'utile discriminante in relazione alla comprensione delle funzioni della struttura. Due esempi di forme poco frequenti.

Gli accumuli realizzati con anfore dal corpo globulare, come le Dr. 20 prodotte nella Betica, ${ }^{96}$ o con quelle a corpo cilindrico di produzione nordafricana, risultano in effetti molto più rari e la forma stessa orienta verso un differente scopo: segnaliamo il caso di Roma, pendici meridionali del

\footnotetext{
${ }^{92} \mathrm{Vd}$. al riguardo Alemani 2008: 10 e per uno sguardo più globale Ibid.: 9-19 e Silva 2008: 43-77..

93 Cfr. ad esempio lo studio delle tipologie anforarie che costituivano la struttura di bonifica rinvenuta a Verona, ex Campo Fiera (Antico Gallina 1996a: 98) e che risultano appartenere alla produzione ispanica di Dr. 7 e Dr. 8 ( Modrzewska, Pianetti 1995: 147-155).

${ }_{94}$ Antico Gallina 1998b: 73-79; 1996a: 84- 89, 94 96, figg. 3,1-3 e 6,1 .

${ }^{95}$ Ead. 1993: 58, 64; 1996a: 106; 1996c e 1998b; 2011 b.

${ }^{96}$ Tipologia ben attestata in Italia durante la prima e media età imperiale.
}

Gianicolo, ${ }^{97}$ relativo alle strutture di un santuario nella fase (fine II sec. d. C.) in cui esso fu adibito al culto di Atargatis, di cui venne rinvenuta una statuetta, e quello dell'horreum ostiense già ricordato.

Se nel primo esempio difficile è, ad oggi, l'interpretazione proprio per la scarsità dei dati stratigrafici e litologici utili in tal senso, non possiamo evitare di rimarcare da una parte l'uso di anfore dal corpo molto espanso e dall'altra l'analogia 'morfologica' della deposizione anforaria con il caso di anfore Mañà C 2 (fine II sec. a. C.) poste anch'esse allineate entro una trincea larga $\mathrm{cm} 35$ scavata nella roccia, parallele alla piattaforma dell'edificio di culto e considerate un elemento di filtraggio in relazione con la sala della piscina del complesso cultuale dedicato ad Atargatis. Situato sulla collina alle spalle dell'estuario del Molinete, a Carthago Nova, ${ }^{98}$ è confrontabile, secondo gli AA., con un altro «filtro di carattere cultuale» individuato ad Ampurias (Neapolis). Ad Ampurias una cisterna rettangolare era bordata da 7 anfore di tipo greco-punico infisse nel suolo; la presenza alla loro base di un foro posto verso l'interno della cisterna non lasciò dubbi a Beltrán Lloris circa l'utilizzazione della struttura anforaria. ${ }^{99}$ Per Carthago Nova non si hanno però indicazioni di analoghi fori di filtraggio dell'acqua.

Il termine del confronto fra i casi si limita, ovviamente, al tipo di posizione 'allineata' delle anfore.

Passando da ciò alla adeguatezza o inadeguatezza della forma ad interventi di bonifica, si è recentemente avuta occasione di domandarci se le anfore Dr.20 del santuario al Gianicolo, coricate e sovrapposte in tre ordini, disposte non in estensione, ma in un'unica fila coerente con l'orientamento del precedente edificio cultuale, non fossero un modo per

\footnotetext{
${ }^{97}$ Pasqui 1909: 403-409. Non disponiamo di elementi sufficienti per avanzare un'ipotesi di interpretazione funzionale delle due tipologie di accumuli, che risultano comunque allineati secondo l'orientamento delle strutture che hanno preceduto quelle del santuario siriaco del IV secolo e che, nella I fase del I sec. a.C.-I sec. d.C., secondo alcuni (Meneghini 1982: 50; Calzini Gysens 1982: 62-64) potrebbero anche non essere di carattere religioso, ma connesse alle attività commerciali svolte nella zona: innumerevoli anfore del tipo ispanico Dr. 7-11 erano infisse verticalmente in uno strato di cenere e carboni, secondo due allineamenti ortogonali e coerenti con i muri del supposto antico santuario; anfore Dr. 20 erano poste orizzontalmente su tre ordini sovrapposti con andamento parallelo ad uno degli accumuli precedenti. Non rientra nella tipologia della bonifica l'accumulo di anfore Dr. 20 rinvenuto nell'ultimo quarto del 1800 negli Orti Torlonia, ai piedi del monte Testaccio, a contenimento della collina formatasi con gli scarichi delle anfore giunte in città. Significativo il fatto che in Roma esistessero commercianti all'ingrosso di olio e vino prodotti nella Betica.

${ }_{98}$ Ramallo Asensio, Riuz Valderas 1994: 79-102.

${ }^{99}$ Beltrán Lloris 1970: 583.
} 
conservarne la memoria in quanto area sacra 'incancellabile', nella sostanza riconducibile alle lagoenae sepolte ricordate dai gromatici come fines sepolturaril $^{100}$ e se a questa ipotesi si potesse accostare il caso ispanico citato, qualora non sia ammissibile una motivazione fondata sulle peculiarità del suolo roccioso su cui poggiava l'edificio templare e sulle sue esigenze. Va osservato, seguendo anche la relazione di Nicole e Darier ${ }^{101}$ e la sezione riportata, che una canalizzazione di mezzi corpi anforari e due allineamenti fra loro ortogonali di anfore oblunghe ${ }^{102}$ poste ritte seguivano - parrebbe alla stessa quota $^{103}$ - l'orientamento dei lacerti murari datati alla fine I sec. a. C. - I sec. d. C., ${ }^{104}$ analogo a quello dei muri del tempio del II sec. d. C. Ad un livello superiore - secondo la sezione di Nicole e Darier- in corrispondenza dell'endonartece del tempio tardo, ritroviamo ancora l'allineamento delle Dr. 20 , orientato secondo la planimetria del vecchio tempio.

Pur considerando la forte incertezza delle relazioni stratigrafiche ${ }^{105}$ cui attribuire gli allineamenti anforari, se, secondo alcuni, il nuovo intervento costruttivo ha previsto la ricolmatura dell' area, includendo «un dispositivo sotterraneo di drenaggio o di prosciugamento del terreno», ${ }^{106}$ non riterrei fugato il dubbio che, in questo caso, non ci si trovi dinnanzi ad una bonifica $\mathrm{o}$ ad un drenaggio, ma ad una modalità sacrale reiterante la planimetria del precedente luogo di culto. Osservando la topografia del sito, la posizione del santuario, in effetti, è al limite fra due unità geologiche di diverso comportamento rispetto all'elemento 'acqua sotterranea': l' unità delle argille azzurre e la stretta fascia di sabbie grigie dell'unità di Monte Mario. Una condizione idrogeologica e una collocazione 'topografica' che si conciliano con la

${ }^{100} \mathrm{Si}$ pensi al terminus orcularis o laguenarius (confine segnato con piccole giare o con brocche), modalità attestata in alcuni brevi trattati gromatici quali Ex libris Dolabellae, in Gromatici veteres, ed . C. Lachmann, Berlin 1848, p. 303, fig. 228 (cacabos [...] aut orcas fractas aut certe integras usati come segnale di fines sepolturarios; a distanza di cinque piedi dalla effettiva sepoltura); Faustus et Valerius VV.PP. Auctores, ibid., p. 308, fig. 247 (a proposito dei termini usati in Africa e in Gallia, si parla di laguinas tres quadrifinium faciunt); Terminorum diagrammata, ibid., p. 341, fig. 300 ( seria $=$ grosso vaso, specie di giarra, di terracotta per contenere vino od olio); Vitalis et Arcadius auctores, ibid., p. 344, fig. $326 \mathrm{G}$.

${ }^{101}$ Nicole, Darier 1909: 28-31.

${ }^{102}$ I materiali non risultano ancora studiati (Calzini Gysens 1982: 61, nota 1). Parrebbe, dalle immagini fotografiche, di poter individuare, fra le altre, anfore ispaniche Dr. 7-8.

103 Nicole, Darier 1909: 28-29.

${ }^{104}$ Steinby $1993: 141$

${ }^{105}$ Lo sottolinea chiaramente Calzini Gysens, 1982: 62.

${ }^{106}$ Steinby 1993: 142. presenza di sorgive ${ }^{107}$ e con le esigenze di una bonifica, ${ }^{108} \mathrm{ma}$, nonostante ciò, ci appare assai problematica la reale comprensione dei 'rigorosi' allineamenti di anfore in questo sito. I caratteri idraulici potrebbero anche far pensare all'esigenza di una sorta di doppia griglia lineare a compensazione di un terreno imbibito e ad una paratia di contenimento della parte di superficie utilizzata per la realizzazione del santuario di II secolo e di salvaguardia dei suoi muri: un caso che morfologicamente ricondurrebbe agli allineamenti usati ad Arles, Ile de Sable, ad esempio, come strutture d'argine o come 'rete' di contenimento su un terreno inondabile. ${ }^{109}$

I dubbi permangono. In effetti, il corpo sferico delle Dr. 20 poste in orizzontale non si prestava a scaricare pesi edilizi sovrapposti quanto le anfore affusolate; più consona all' ampio corpo 'pieno d'aria' la funzione di bonifica idraulica (struttura di aerazione, di isolamento dall'umidità per area aperta frequentata), o di riempimento consolidante di un terreno cedevole (ma perché, allora, in file così regolari?). Ne' sono mai chiari nella letteratura archeologica i valori delle pendenze, che potrebbero conciliarsi con l'uso degli accumuli per abbassare il livello della falda qualora la canalina citata fosse il 'dreno' collegato con il sistema ad anfore, quindi una bonifica, ${ }^{110} \mathrm{o}$ qualora la lunghezza degli accumuli rispondesse esattamente alla formula che oggi applichiamo per valutare il rapporto 'pendenza-lunghezza drenaggio', - concettualmente ovvia per l'empeiría degli antichi- in un' area che restò cortilizia scoperta e di accoglienza dei fedeli ${ }^{111}$ nella riedificazione del IV secolo (Fig. 12).

A ben vedere la presenza di anfore Dr. 20 pare connotare scelte costruttive connesse con l'esigenza di salvaguardare muri vicino ai quali furono poste dalla trasmissione di umidità e, contestualmente, di sostenere terreni che potrebbero mettere in pericolo la stabilità delle murature.

Dai numerosi casi della Narbonense ricordati e studiati anche da Laubenheimer ${ }^{112}$ rimarco il fatto che

${ }^{107}$ Cfr. Mocchegiani Carpano 1982: 39-40 e fig. 1, A, B.

108 Marra, Rosa, 2004: unità geologiche nn. 18 (sabbie grigie) e 19 (argille azzurre).

${ }_{109}$ Laubenheimer 1998: 50-52

${ }^{110}$ Il fondo-pozzo indagato a monte dell' area di scavo da Gauckler era a quota m 51 s.l.m., pari a m 7,10 dalla superficie topografica, quindi la prima falda antica doveva essere in quel punto intorno a $\mathrm{m} 52,50 \mathrm{~s} .1 \mathrm{~m}$. Si sa che il santuario era collocato in un 'anfiteatro naturale' fra le quote 45 e 55 s.l.m. (Meneghini 1982: 47).La canalina laterizia smaltiva in direzione E (Nicole, Derier 1909, pl. XII, p-q-r), ma non sono percepibili i rapporti stratigrafici.

111 Meneghini 1982: 50, 52.

${ }^{112}$ Laubenheimer 1998: 54-68. 


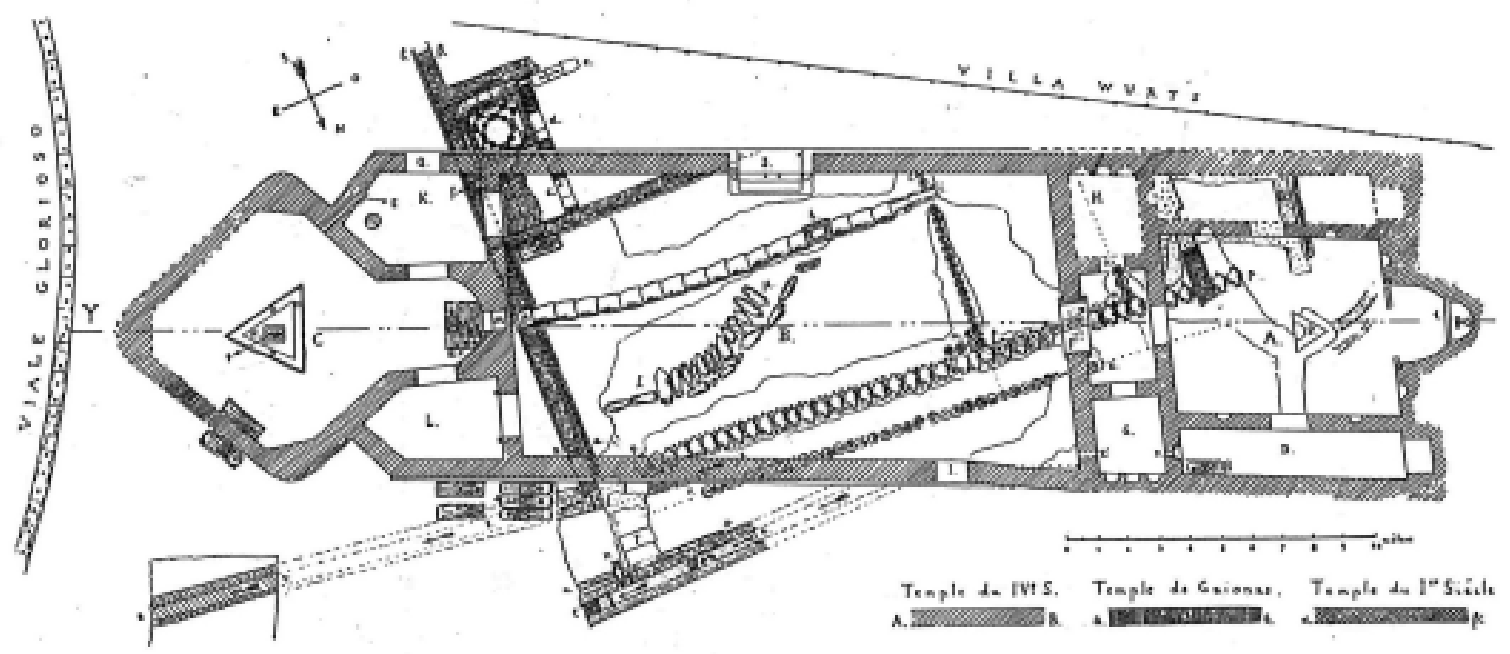

Figura 12. Roma, il santuario siriaco, pianta integrata dagli esiti degli scavi 1981-82 (da L'area del 'santuario siriaco del Gianicolo').

alle Dr. 20 spesso non si sovrapponessero murature, ma le affiancassero e la compresenza di ghiaia e/o sabbia mi pare concorra a riprovare - con la loro elevata permeabilità all' aria- la metodica di una bonifica idraulica con funzione di struttura di aerazione per la salubrità del vicino muro o, più raramente, dell'ambiente sovrastante. La sottrazione dell'acqua capillare avviene, lo si deve ribadire, non per gravità — come nel caso del drenaggio- ma attraverso i meccanismi dell'evaporazione - come nel caso delle strutture di aerazione-, favorita dalla libera circolazione dell' aria e degli elementi a contatto con l'accumulo. ${ }^{113}$

Uno degli accumuli rinvenuti ad Aquileia e ritenuto un drenaggio, ${ }^{114} \mathrm{mi}$ sembra invece 'parlante' più dal toponimo - località Palude di S. Antonio_— o dalla immagine a corredo dell' articolo che non dalla definizione: anfore Dr. 20, in doppia fila, capovolte per un lungo tratto, parallele al cardo maximus urbano. Dove stanno gli elementi a supporto dell'ipotesi 'dreno'? Quali le caratteristiche del terreno a contatto con le anfore? Non è più probabile si tratti di bonifica geotecnica a sostegno di una fascia di terreno cedevole, in relazione alla non distante strada?

In merito, poi, alla tipologia di anfora a corpo cilindrico di produzione africana riscontrata nell'esempio ostiense, ma oggettivamente poco frequente nei sistemi di bonifica, ne sottolineiamo la maggiore e più nota diffusione come materiale di alleggerimento nelle volte delle grandi opere architettoniche tardoantiche.

\footnotetext{
${ }^{113}$ Antico Gallina 1996a: 80-81.

${ }^{114}$ Maselli Scotti 1998: 107-111.
}

In generale risulta, negli studi, ancora prevalente un'attenzione focalizzata sul carattere morfologico dei contenitori, tesa a privilegiare l'aspetto cronologico dei manufatti più che quello tecnico-funzionale. E' evidente che le strutture di bonifica ad anfore rappresentano, come pure le sepolture in anfora, dei contesti 'chiusi' e, soprattutto per le prime menzionate, sicuramente omogenei, contesti in cui l'elemento datante è certo fondamentale, ma possono prestarsi ad altre considerazioni.

Ad esempio un intervento di 'bonifica sulla bonifica', cioè un riadattamento, una modifica, un rifacimento dell'originaria opera geotecnica: è il caso del quartiere suburbano occidentale di Mediolanum, ove un intervento di restauro di un precedente accumulo anforario fu realizzato con la posa di un nuovo livello di contenitori capovolti. ${ }^{115}$

Senza contare che la stessa originaria opera geotecnica era realizzata prevalentemente riutilizzando manufatti successivamente al momento in cui il loro ciclo vitale (conservazione, trasporto) si era concluso, almeno all'interno dei circuiti commerciali, per così dire, 'di superficie'. ${ }^{116}$ Iniziava in seguito per le anfore un diverso e nuovo ambito di utilizzo, mi sia consentito, 'ipogeo', per il quale è anche legittimo pensare ad un eventuale scarto temporale fra datazione della tipologia anforaria e datazione della struttura ad anfore quale 'momento del reimpiego'. La quantificazione dello scarto cronologico è offerta

\footnotetext{
115 Bruno 1998: 262 e fig. 1.

${ }^{116}$ L'utilità di sottoporre ogni anfora costituente l'accumulo ad analisi è indiscutibile, a fronte di rari casi di indagine dei residui visibili.
} 
dalla relazione stratigrafica 'struttura ad anfore -opera edilizia' e dalla relazione 'tipologia anforemateriale interstiziale'.

Un altro problema non ancora approfondito, ma stimolante, potrebbe essere quello degli eventuali circuiti commerciali riservati o meno a questo secondario uso del materiale anforario che ebbe a prediligere le forme affusolate: ${ }^{117}$ entrerebbero nuovamente in gioco i luoghi di produzione dei manufatti d'uso ed i canali commerciali seguiti per la funzione originaria, la prima funzione o, se vogliamo, la prima vita (contenitori di derrate alimentari), con la quale potrebbero confrontarsi i dati desumibili dalle attestazioni della seconda funzione o della seconda vita (materiale edilizio per la bonifica dei suoli) e dei luoghi ove avvenne non solo lo smercio, ma il riuso e l'eventuale suo restauro o modifica. Si ritiene che questa ipotesi di lavoro rappresenti una strada percorribile, da cui potrebbe trarre luce anche la problematica relativa alla trasmissione delle conoscenze tecniche e degli ambiti d'applicazione.

Pensiamo che anche la verifica della dislocazione — urbana e/o rurale — degli accumuli anforari nella più vasta geografia delle attestazioni potrebbe rappresentare la spia di un percorso culturale del modello di bonifica applicato ai suoli problematici e del ruolo assunto, per 1'Hispania ad esempio, dalle città della Tarraconensis e dal grande fiume Ebro. ${ }^{118}$ In tal senso la collaborazione da parte degli archeologi e topografi spagnoli rappresenterebbe un'insostituibile occasione di censimento, di studio e di confronto, cui mi auguro si possa giungere, anche dietro la sollecitazione di questa breve nota e delle possibili mie manchevolezze relativamente alla documentazione ispanica. $^{119}$

Altro problema che non mi pare sia stato ancora posto in essere è legato alla sfera giuridica e religiosa dell' atto costruttivo, problema che impone il confronto fra archeologi, storici del diritto e storici delle religioni e che si è posto all'osservazione in un recente Convegno su I riti del costruire nelle acque violate. $^{120}$

117 Rare, infatti, le strutture realizzate con anfore Dr.20, come si è detto. Non attestato l'uso di anfore con peduncolo a bottone, come nei tipi Dr. 25, ad esempio, se non in ancor più rari casi (Novaria).

${ }^{118}$ L'importanza del fiume è ben delineata in Beltràn 1961: 65-79.

${ }^{119}$ A questo proposito riterrei ugualmente interessante confrontarsi anche sull'uso sperimentale, in futuri scavi di accumuli anforari, della Scheda elaborata da chi scrive e pubblicata in Antico Gallina 2008b: 21-42; 2011a.

${ }^{120}$ Antico Gallina 2010: 295-315 e i restanti numerosi saggi. Fra essi rammento il contributo di Ribera i Lacomba 2008: 269-292 e in particolare 278 .
Non possiamo, comunque, affermare di trovarci di fronte ad un'invenzione della tecnica costruttiva romana, né ad una prerogativa del suolo italico, ma furono sicuramente i Romani a reiterare la metodica e ad esprimere il loro ingegnoso spirito di rielaborazione nella diffusione che soprattutto fra I sec. a. C. e I sec. d. C. ${ }^{121}$ ebbe il sistema applicato agli interventi nei terreni idromorfi o imbibiti destinati, comunque, ad opere edilizie. ${ }^{122} \mathrm{Che}$, dunque, questo aspetto applicativo possa rientrare nelle politiche urbanistiche dell'età di Augusto è ammissibile.

Da uno sguardo più dilatato possiamo presumere il ruolo protagonista dell'Oriente estremo e, in seconda battuta, del vicino Oriente coloniale greco, sulla quinta scenografica di un Mediterraneo mediatore di culture e di idee, strumento primario di comunicazione fra i popoli, elemento non di distacco, ma di commistione. ${ }^{123}$ Il mondo insulare egeo (Rodi, Thasos, Kea) dal IV sec. a. C., gli ambiti coloniali greci, come la dacica Histria (prima metà del v sec. a. C.), Massalia (prima metà del IV sec. a. C.), ${ }^{124} \mathrm{Me}-$ taponto (seconda metà del IV sec. a. C.), la Atene ellenistica e ancora Tripoli (III-II sec. a. C.) e Cartagine (età augustea) ${ }^{125}$ rappresentano una sorta di file rouge della diffusione di un sistema — quello dell'uso alternativo di anfore- che radica nel mondo orientale se, come mi è parso, ne è ravvisabile uno dei possibili modelli nella Korsabad del regno dei Sargonidi (vII sec. a. C.). ${ }^{126}$

${ }^{121}$ Antico Gallina 1996a: 71.

${ }^{122}$ Ibid.: 71: la rarefazione della tecnica si osserva dal II sec. d. C. Sempre più rari i casi posteriori al III secolo, dei quali ricordo quelli di Ostia (III sec. d. C.), di Roma (III sec. d. C. e periodo successivo), di Calvatone e di Torino (III-IV sec. d. C.), di Ravenna (v-vi sec. d. C.). Dalla media età imperiale è più facile individuare sistemi a pali come opere di bonifica a scopi fondazioniali, mentre le anfore si ritrovano usate soprattutto come elementi di alleggerimento nelle volte delle grandi opere architettoniche, prassi, comunque, già nota da lungo tempo. Nella ricerca di giustificazioni va soppesato non solo la diversa direzione di mercato dei prodotti di consumo e dei relativi contenitori, ma anche, ad esempio, le differenze di gusto e di stile di vita dimostrate anche dal fenomeno della contrazione dell'area urbana, di rioccupazione e di riconversione di spazi pubblici e privati, più che di nuove edificazioni, e da quello del commercio di materiali di spoglio, rispondente al gusto, ora prevalente, per l'accostamento fra materiali di diverso pregio.

${ }^{123}$ Antico Gallina 1996a: 104; Ead. 2006: 94.

${ }^{124}$ Nella zona portuale, un letto di anfore marsigliesi era funzionale non certo ad un drenaggio, ma al consolidamento del terreno instabile che doveva essere interessato da un asse stradale: per la descrizione del ritrovamento vd. Guery 1992: 112. Per situazioni assimilabili meritevoli di verifiche vd. le descrizioni di strutture portuali in Bernal, Saez et alii 2005: 214 ss., tratte da Bonifiche e drenaggi.

${ }^{125}$ Struttura di sostegno e di contenimento. Vd. Ladjimi Sebaï 2005: 84-85.

${ }^{126} \mathrm{E}$ ' possibile che gli esempi di sistemi costruttivi che si avvalevano di vasi ovoidali o di altri analoghi oggetti cerami- 
Il sistema di bonifica geotecnica o idraulica non vide modificarsi nei secoli il criterio informatore, ma solo la sostituzione del tipo di materiale: nei tempi moderni non più anfore, ovviamente. Non solo ciottoli o ghiaia, ma argilla espansa, polistirolo, lana di vetro ed altri materiali da costruzione leggeri esercitano oggi le stesse funzioni delle anfore dell'antichità, costituendo il sottofondo o la fondazione di strutture pesanti oppure l'isolamento dall'umidità capillare ed altro ancora.

\section{BIBLIOGRAFIA}

AA.VV. 1984: El forum Romá d'Empúries, Barcelona. Acque interne 1996: Acque interne: uso e gestione di una risorsa, M. Antico Gallina (ed.), Itinera. I percorsi dell'uomo dall'antichità ad oggi, 1, Milano.

Alemani, P. 1996: «Lineamenti geologici ed idrogeologici della Pianura Padana», Acque interne, 928.

Alemani, P. 2008: «Descrizione dei suoli: significati, pratica, adozione di standard tecnici», Terre terreni territori, 9-19.

Antico Gallina, M. 1982: «A proposito del confine settentrionale del municipium di Mediolanum», Sibrium XVI, 133-147.

Antico Gallina, M. 1993: «L'assetto territoriale di Mediolanum: proposta di lettura», Civiltà padana. Archeologia e storia del territorio, IV, 51-88.

Antico Gallina, M. 1996a: «Valutazioni tecniche sulla cosiddetta funzione drenante dei depositi di anfore», Acque interne, 67-112.

Antico Gallina, M. 1996b: «Il rapporto città-campagna. Brevi riflessioni, Milano in età imperiale. IIII secolo», Atti del Convegno di Studi (Milano, 1992), Milano, 99- 106.

Antico Gallina, M. 1996c: «Mediolanum, città d'acqua: i ponti», Strade romane, ponti e viadotti, ATTA 5, 195-212.

Antico Gallina, M. 1997a: «Elementi di topografia urbana. I pozzi di Mediolanum: struttura e funzione», Rassegna di studi del Civico Museo Archeologico e del Civico Gabinetto Numismatico LIXLX, 127-154.

ci siano numerosi: la ricerca in questo senso comporterebbe indagini più estese rispetto a quelle fatte da chi scrive, nelle quali ci sia il concorso di archeologi orientalisti. Il censimento bibliografico non è comunque facile, dal momento che anche per l'ambito greco e per l'ecumene romano la difficoltà del reperimento dei dati è aumentata dalla non conoscenza delle finalità del sistema e quindi da una scarsa attenzione a questo tipo di ritrovamento, spesso segnalato per la sola tipologia delle anfore.
Antico Gallina, M. 1997b: «Regolamentazione idrica nel suburbium di Mediolanum», Atti del Convegno «Uomo, acqua, paesaggio» (S. Maria Capua Vetere, 1996), ATTA, Suppl. 2, Roma, 355-359.

Antico Gallina, M. 1998a: «Le anfore come elemento funzionale a interventi di bonifica geotecnica e idrogeologica: alcune riflessioni», Bonifiche $e$ drenaggi, 73-80.

Antico Gallina, M. 1998b: I Romani dalla terra all'uomo, Popoli dell'Italia antica, 7, Cinisello Balsamo.

Antico Gallina, M. 2000: «Sui sistemi di riutilizzo delle anfore. I casi connessi con l'edilizia», Rendiconti dell'Istituto lombardo. Classe di Lettere e Scienze Morali e Storiche 199 (1999), fasc. 1, 7596.

Antico Gallina, M. 2002: «Peculiari tecniche di miglioramento dei terreni di fondazione usate dai costruttori romani in presenza di acqua ipogea», Rendiconti dell'Istituto lombardo. Classe di Scienze Chimiche e fisiche, Geologiche, Biologiche e Mediche 134 (2000), fasc. 1-2, 252-274.

Antico Gallina, M. 2004a, «Le pompe idrauliche di Mediolanum: dalle particolarità costruttive alle relazioni con i caratteri ambientali», Problemi di macchinismo in ambito romano. Macchine idrauliche nella letteratura tecnica, nelle fonti storiografiche e nelle evidenze archeologiche di età romana, F. Minonzio (ed.), Atti della Giornata di studio (Como, 2002), Como, 125-142.

Antico Gallina, M. 2004b: «Fra utilitas e salubritas: esempi maltesi di bonifica con strutture ad anfore, Acque per l'utilitas, per la salubritas, per l'amoenitas», M. Antico Gallina (ed.), Itinera. I percorsi dell'uomo dall'antichità ad oggi 4-5, Milano, 245-271.

Antico Gallina, M. 2006: «Lo sfruttamento delle acque ipogee e la loro gestione: dall'oriente all'occidente, attraverso Roma, tre esempi di trasferimenti tecnologici nella lunga durata», M. Mergier (ed.), Transferts de technologies en Méditerranée (Montecatini, 2001), Paris Sorbonne, 87-102.

Antico Gallina, M. 2008a: «Bonificare il suolo: le strutture ad anfore rinvenute ad Augusta Praetoria (Aosta)», Terre, terreni, territori, 79-100

Antico Gallina, M. 2008b: «Per la schedatura delle strutture ad anfore: elaborazione di una proposta», Terre, terreni, territori, 21-42.

Antico Gallina, M. 2008c: «Munire viam: un significato ammissibile», Rendiconti dell'Istituto lombardo. Classe di Lettere e Scienze Morali e Storiche 142, 371-396. 
Antico Gallina, M. 2010: «Sistemi ad anfore per la bonifica nei terreni di fondazione: una sacralità disattesa?», H. Di Giuseppe, M. Serlorenzi (edd.), Atti del Convegno Internazionale su I riti del costruire nelle acque violate (Roma giugno 2008), Roma, 295-315.

Antico Gallina, M. 2011a, «Bonifiche geotecniche e idrauliche con anfore: teoria e pratica di un fenomeno», www.fastionline.org/docs/FOLDER-it2011-226.pdf., 1-22.

Antico Gallina, M. 2011b, «Archeologia del legno. Uso, tecnologia, continuità in una ricerca pluridisciplinare», Milano.

Antico Gallina, M. 2011c, «Si vero furno[...] cum caccabos fecerit» (Mem. 7 A). Spunti per la rilettura di una tecnica nella lunga durata», Archeologia dell'architettura, XV, c.d.s.

l'area 1982: l'area del 'santuario siriaco del Gianicolo'. Problemi archeologici e storico-religiosi, Roma.

Barbieri M. E., Manzella V. 2006: Emilia-Romagna, Coll. Archeologica delle Regioni d'Italia, Roma.

Beltrán, A. 1951: «Interesante pieza escultorica hallada resientemente en una «villa» romana de Zaragoza», AEspA 82, 497-506.

Beltrán, A. 1961: «El rio Ebro en la Antigüedad Clásica», Caesaraugusta 17-18, 65-79.

Beltrán Lloris, M. 1970, Las anforas romanas en España, Zaragoza.

Beltrán Lloris, M. 1991: «El patrimonio arqueológico de Caesaraugusta: Descubrimiento e incorporatción a la historia de Zaragoza», Caesaraugusta 68, 77- 150.

Bernal D., Sáez, A. M., Montero R., Díaz J. J., Sáez A., Moreno D., Toboso E., 2005: «Instalaciones fluviomarítimas de drenaje con ánforas romanas: a propósito del embarcadero flavio del caño de Sancti Petri (San Fernando, Cádiz)», SPAL 14, 179-230.

Bishop. J., Mariotti Branca, V. 1985: «Cremona, via Amidari Bissolati», Notiziario della Soprintendenza Archeologica della Lombardia, 116-118.

Bonifiche e drenaggi 1998: Bonifiche e drenaggi con anfore in epoca romana: aspetti tecnici e topografici, Atti del Convegno, S. Pesavento Mattioli (ed.) (Padova, 19-20 ottobre 1995), Modena.

Bruhl 1964: «Informations Archéologiques. Circoscription de Lyon», Gallia 22, 413.

Bruno, B. 1998: «Le strutture di bonifica con anfore rinvenute a Milano nello scavo dell'Università Cattolica», Bonifiche e drenaggi, 259-264.

Calvani Marini, M. 1992: «Strade romane dell'Emilia occidentale», Tecnica stradale romana, ATTA 1, 187-192.
Calvani Marini, M. 1998: «Banchi d'anfore nell'Emilia occidentale», Bonifiche e drenaggi, 239-252.

Calzini Gysens, J. 1982: «Osservazioni sulle fasi I e II del santuario con riferimento alle ultime campagne di scavo», L'area del 'santuario siriaco del Gianicolo, 61-71.

Catarsi Dall'Aglio, M. 1998: «Diversi tipi di bonifica in alcune ville romane del Parmense», Bonifiche e drenaggi, 253-258.

CAR 1977: Carta archeologica di Roma, Firenze.

Cebolla Berlanga, J. L., Dominguez Arranz, A., Ruiz Ruiz, F. J., 2004: «La excavación arqueológica del solar de la plaza de Las Tenerías, $n^{\circ}$ 3-5 (Zaragoza)», Salduie 4, 463- 472.

Coarelli, F. 1984: Guida archeologica di Roma, Roma (ristampa).

Dall'Aglio, P. L. 1992: «Acciottolati stradali di età romana e rete viaria principale nel territorio reggiano», Tecnica stradale romana, ATTA 1, 179-186.

Delattre, R. P. 1894: «Le mur à amphores de la coline Saint- louis a Carthago», Bull. Arch. du Com. des travaux historiques et scientifiques, 89-119

Domínguez-Arranz, A., Vallejo Rasero, J. M., Ruíz Llera, S. 2003: «Las ánforas de Bibracte en época romana: tipología y epigrafía», Salduie 3, 83-91.

Dressel, H. 1879: «Un grande deposito di anfore rinvenuto nel nuovo quartiere del Castro Pretorio», Bull. Comm 7, Roma, 36-112.

Guery, R. 1992: «Le port antique de Marseille», Marseille grecque et la Gaule, Actes du Colloque

International d'histoire et d'archéologie et du V Congrès Archéologique de la Gaule méridionale (Marseille 1990), Travaux du Centre Camille-Jullian 11, 109-121.

Jorio, S., Donati, M. T., Masseroli, S., Perani, G., Tibiletti, T. 2005: Dalla rimozione della memoria alla riscoperta. Indagini archeologiche a Laus Pompeia - Lodi Vecchio, Catalogo della Mostra, Milano.

Ladjimi Sebaï, L. 2005: «La colline de Byrsa à l'époque romaine. 'Etude épigraphique et état de la question», Karthago XXVI, 84-85.

Laubenheimer, F. 1998: «L'eau et les amphores. Les systemes d'assainissement en Gaule romaine», Bonifiche e drenaggi, 47-70.

Lostal Pros, J. 1978: Arqueología del Aragón Romano, Caesaraugusta, 45-46, 67-112

Lugli, G. 1957: La tecnica edilizia romana con particolare riguardo a Roma e Lazio, vol. 2, Roma.

Mapa geológico de la Ibérica, Baleares y Canarias 1994. Madrid.

Mar, R. (ed) 1993: Els moments provincials de Tárraco. Noves aportacions al seu coneixement, Documents d'arqueologia classica 1 Tarragona. 
Mariotti, V. 1998: «Cremona. Il drenaggio con anfore di via Amidani/Bissolati», Bonifiche e drenaggi, 291-292.

Marra, F., Rosa, C. 2004: Carta geologica del centro di Roma, Roma.

Maselli Scotti, F. 1998: «Bonifiche e drenaggi con anfore ad Aquileia», Bonifiche e drenaggi, 107111.

Meneghini, R. 1982: «Il 'santuario siriaco del Gianicolo'. L'impatto architettonico», L'area del 'santuario siriaco del Gianicolo', 47- 59.

Mocchegiani Carpano, C. 1982: «Le sorgenti», L'area del 'santuario siriaco del Gianicolo', 39- 43.

Modrzewska, I., Pianetti, F. 1995: «Anfore spagnole del deposito di Verona (Italia). Interpretazione dei dati analitici», AEspA 67, 147-155.

Morel, Ch. 1877, Amphora, Ch. Daremberg, E. Saglio, Dictionnaire des antiquités grècques et romaines d'après les textes et les monuments, I, Paris.

Nicole, G, Darier, G. 1909: «Sanctuaire des dieux orientaux au Janicule», Mélanges d'arcchéologie et d'histoire XXIX, 3-86.

Pasqui, A. 1909: «Roma. Nuove scoperte nella città e nel suburbio», NScavi, s.V, 389-410.

Passi Pitcher L. 1994: «Cremona, via Massarotti. Banco di anfore», Notiziario della Soprintendenza per $i$ Beni Archeologici della Lombardia, 149.

Passi Pitcher, L. 1998a: «La colonia latina di Cremona: vie di comunicazione alla luce dei recenti ritrovamenti», G. Sena Chiesa e E. A. Arslan (edd.), Optima via. Atti del Convegno internazionale di studi «Postumia. Storia e archeologia di una grande strada romana alle radici dell'Europa», (Cremona 1996), Milano, 99-110.

Passi Pitcher, L. 1998b: «Cremona e i banchi d'anfora: riflessioni sulla topografia storica», Bonifiche e drenaggi, 133.

Passi Pitcher, L. 2003: «Archeologia della colonia di Cremona: la città e il territorio», P. L. Tozzi (ed.), Storia di Cremona. L'età antica, Cremona, 130229.

Poggi, F. 1897: Le fognature di Milano. Rapporto dell'Ufficio tecnico Municipale sui lavori eseguiti a tutto l'anno 1897, Milano.

Ramallo Asensio, S. F., Ruiz Valderas E. 1995: «Un edìculo republicano dedicado a Atargatis en Carthago Nova», AEspA 67, 79-102.

Ribera i Lacomba, A. 2008: «Depositos rituales de Valentia (Hispania). De la primera fundación republicana (138 a. C.) a la segunda augustea», H. Di Giuseppe, M. Serlorenzi (edd.), Atti del Convegno Internazionale su I riti del costruire nelle acque violate (Roma giugno 2008), Roma, 269294.

Ridolfi, G. 2006: «Lodi Vecchio (LO). Via don Milani. Contesto pluristratificato», Notiziario della soprintendenza per i beni Archeologici della Lombardia, 94-96.

Ruiz de Arbulo Bayona, J. 2001: «El templo del foro de Ampurias y la evolución de los foros republicanos», Templos romanos de Hispania. Cuadernos de arquitectura romana 1, 11-37.

Salsarola, D., Locatelli, D., Bonetti, C., Colombo, M. E., Matteini, R. 2001: «La campagna di scavo del 1997-98 nell' area dell' antico brolo (UC VIII)», M. Sannazaro (ed.), La necropoli tardoantica. Ricerche archeologiche nei cortili dell'Università $\mathrm{Ca}$ ttolica, Atti delle giornate di studio (Milano, gennaio 1999), Milano, 9-16.

Sanmarti' i Grego, E. (ed.) 1984: El fórum romá d'Empúries (Excavacions de l'any 1989).Una aproximació arqueológica al procés hisóric de la romanitzaciò al nord-est de la penísula Ibérica, Barcelona.

Silva, S. 2008, «I caratteri dei suoli agrari», Terre terreni territori, 43-77.

Steinby, E. M. (ed.) 1993: Lexicon Topographicum Urbis Romae, I, Roma.

Ted'A 1989: «El foro provincial de Tarraco, un complejo arquitectónico de época flavia», AEspA 62, 141-191.

Terre, terreni, territori 2008: Terre, terreni, territo$r i$, M. Antico Gallina (ed.), Itinera. I percorsi dell'uomo dall' antichità ad oggi 6, Milano.

Tirelli, M., Balista, C., Gambacurta, G., Ravagnan, G. L. 1988: «Altino (Venezia): proposte di articolazione in fasi della necropoli «Le Brustolade» attraverso l'analisi di un settore ( trincea I 198587)», Quaderni di Archeologia del Veneto 3, 348394.

Tra terra e acque 2004: G. Spagnolo Garzoli, F. Gambari (eds)., Tra terra e acque. Carta archeologica della Provincia di Novara, Novara.

Volontè, M. 1998: «L'importazione di oggetti d'uso e di pregio a Cremona romana. Spunti dai materiali del Museo Civico «Ala Ponzone» di Cremona», Optima via, 301-310.

Zevi, F. 1972: «Ostia (Roma). Scoperte archeologiche effettuate nei mesi di Settembre e ottobre 1968 nell'Isola Sacra, presso la sponda della Fiumara grande tra il ponte della Scafa e l'ansa», N. Sca$v i$, s.VIII, XXVI, 404-431.

Recibido el 28/04/10

Aceptado el 29/09/10 